\title{
Nononcogenic restoration of the intestinal barrier by E. coli-delivered human EGF
}

\author{
Mira Yu, ${ }^{1}$ Juil Kim, ${ }^{1}$ Jung Hoon Ahn, ${ }^{2}$ and Yuseok Moon ${ }^{1,3}$ \\ 'Laboratory of Mucosal Exposome and Biomodulation, Department of Biomedical Sciences, Biomedical Research Institute, \\ Pusan National University, Yangsan, South Korea. ${ }^{2}$ Korean Science Academy of KAIST, Busan, South Korea. ${ }^{3}$ College of \\ Information and BioMedical Engineering, Pusan National University, Yangsan, South Korea.
}

\begin{abstract}
Although mucoactive proteins, such as epidermal growth factor (EGF), could improve clinical outcomes of intestinal ulcerative diseases, their gastrointestinal application is limited because of their proteolytic digestion or concerns about tumor promotion. In the present study, ATP. binding cassette $(A B C)$ transporter-linked secretion of human EGF from probiotic Escherichia coli (EGF-EcN) was created to promote beneficial actions of the EGF receptor, which is notably attenuated in patients with intestinal ulcerative injuries. Preventive and postinjury treatment with EGF-EcN alleviated intestinal ulcers and other readouts of disease severity in murine intestinal ulcer models. EGF-EcN administration promoted the restitutive recovery of damaged epithelial layers, particularly via upward expansion of highly proliferating progenitor cells from the lower crypts. Along with the epithelial barrier benefit, EGF-EcN improved goblet cell-associated mucosal integrity, which controls the access of luminal microbiota to the underlying host tissues. Despite concern about the oncogenic action of ECF, EGF-EcN did not aggravate colitis-associated colon cancer; instead, it alleviated protumorigenic activities and improved barrier integrity in the lesions. All findings indicate that probiotic bacteria-based precision delivery of human EGF is a promising mucosal intervention against gastrointestinal ulcers and malignant distress through crypt-derived barrier restoration.
\end{abstract}

\section{Authorship note: MY and JK contributed equally to the study. \\ Conflict of interest: The authors have declared that no conflict of interest exists.}

Copyright: (c) 2019 American Society for Clinical Investigation

Submitted: September 26, 2018 Accepted: July 26, 2019 Published: August 22, 2019.

Reference information: /CI Insight. 2019;4(16):e125166. https://doi. org/10.1172/jci.insight.125166.

\section{Introduction}

Upon exposure to ulcerogenic or necrotizing insults, the gastrointestinal mucosa develops characteristic morphological, ultrastructural, and functional changes that reflect the disruption of the mucosal barrier $(1,2)$. Intestinal epithelial cells form a biochemical and physical barrier against the immunogenic luminal environment, and the collapse of epithelial integrity is thus associated with intestinal mucosal disorders, including inflammatory bowel disease and epithelial cancer $(3,4)$. Healing of mucosal erosion in response to deep mucosal injuries requires reconstruction (reepithelialization) of the surface glandular epithelial structures and the lamina propria, including the mucosal microvascular network, nerves, and connective tissue cells, to fill the mucosal defect $(2,5)$. Most events in repair processes in response to ulcerative injuries are controlled by a variety of human growth factors that can modulate extracellular matrix formation, cell migration, and cell differentiation depending on the context of the injury and the tissue-specific conditions $(6,7)$. The gastrointestinal mucosa has a remarkable ability to repair damage with the support of the epidermal growth factor (EGF), which stimulates cell migration and extracellular matrix formation $(8,9)$. Intravenous treatment with EGF protein in gastric ulcer patients is effective at triggering ulcer healing (10). Moreover, an oral trial with high doses of recombinant human EGF enhanced the process of wound repair in the gastrointestinal tracts of patients $(11,12)$. However, less than $1 \%$ of protein drugs administered orally arrive at the target because of their instability and degradation in the digestive tract (13). A protected and enhanced delivery system is required to overcome the problem associated with the application of oral recombinant therapeutics for intestinal ulcerative disease.

Among various protein drug delivery systems, intestinal commensal bacteria or probiotics can carry and deliver key medicinal components to injured epithelial targets, such as those in patients with intestinal ulcerative diseases, including inflammatory bowel disease $(14,15)$. Genetically modified probiotics might be a good alternative to mucosal biotherapy as a live, safe carrier. Escherichia coli strain Nissle 1917 $(\mathrm{O} 6: \mathrm{K} 5: \mathrm{H} 1, \mathrm{EcN})$ is a nonpathogenic fecal isolate that has been used as a probiotic agent in human and 
animal medicine to treat chronic inflammatory and infectious diseases of human and animal intestines (16-18). Moreover, limited clinical investigations using EcN and other microorganisms have demonstrated that probiotic-based therapeutic application can be efficacious in patients with chronic ulcerative colitis (19, 20) and irritable bowel syndrome (21). EcN is relatively safe for therapeutic applications because it does not cause colitis, even in gnotobiotic animals monoinoculated with the strain (22). In terms of molecular genetics, $\mathrm{EcN}$ does not produce any virulence factors or carry any genes for pathogenicity traits, and it does not form enterotoxins, cytotoxins, or hemolysins $(23,24)$, all of which support the general recognition of $\mathrm{EcN}$ as a safe organism for human use.

In our previous study, EcN was transiently constructed to secrete human EGF in conjunction with the lipase ATP-binding cassette $(\mathrm{ABC})$ transporter recognition domain (LARD) via the $\mathrm{ABC}$ transporter and then tested for its healing actions in vitro in physical wounds in cultured cells (25). The results suggested that recombinant bacteria had potential for use as a therapeutic agent against intestinal ulcerative and associated malignant diseases. In the present study, a bacterial ABC transporter-based secretion system was stably encoded in the bacterial chromosome as a microbial factory to facilitate the protective and prolonged actions of mucoactive EGF in the gut of experimental animals with intestinal ulcerations and malignancy as a preclinical evaluation. Moreover, assessment of the intervention in the acute and malignant niche would provide biological evidence of the safe actions of mucosal EGF from the probiotic secretion, which is a useful delivery cassette for protein therapeutics in the gastrointestinal tract.

\section{Results}

Prolonged colonization of EGF-EcN in the murine gut. To address the effects of EGF in patients with inflammatory bowel disease (IBD), EGF receptor (EGFR) expressions were compared using 3 IBD-based data sets. The data sets consisted of gene profiles from different types of IBD, including the colon-only Crohn's disease (CD), ileocolonic CD (iCD), and ulcerative colitis (UC). Compared with the EGFR level in the healthy control, its expressions in patients with CD and UC were significantly downregulated (Figure 1A). In particular, tissue-specific expressions in the colon (Vimeire's data set, GEO ID GSE75214) and ileum (Haberman's data set, GEO ID GSE57945) showed significant suppression of EGFR expression in patients with lesions. Reduced expression of EGFR indicates the gastrointestinal attenuation of EGFR-associated actions in patients with IBD. Thus, it can be hypothesized that supplementation with external EGF protein would counteract clinical outcomes by enhancing ligand-activated, EGFR-mediated pharmacological actions despite reduced EGFR levels in patients. Aimed at the human EGFR of patients, the recombinant probiotic EGF-EcN was created by introducing genes encoding a human mature EGF polypeptide linked to 1 fragment of LARD3 and prtDEF, an ABC transporter of Erwinia chrysanthemi, into the EcN chromosome via recombination at the OmpC gene (Figure 1B). The prtDEF is composed of 3 functional units: outer membrane protein (prtF), membrane fusion protein (prtE), and ATP-binding cassette (prtD). In particular, LARD3 binds to the intracellular part of prtDEF, which guides the unfolded peptide to the transmembrane protein complex. Although human EGF is only about $80 \%$ homologous with mouse EGF (25), it strongly activated mouse EGFR (Supplemental Figure 1A; supplemental material available online with this article; https://doi.org/10.1172/jci.insight.125166DS1). Based on this biochemical evidence, we assessed murine responses to human EGF-secreting EcN as a preclinical evaluation.

Before application to the mammalian gut, the carrier bacteria (EGF-EcN) was tested for its colonization activity in the gut of Caenorhabditis elegans (Figure 1C). The control E. coli OP50 handed over most of the gut territory to the mucoadherent EPEC. Compared with the control E. coli OP50, EGF-EcN showed successful colonization activity in the gut of $C$. elegans in the presence of a competitor EPEC. Next, the recombinant bacteria were assessed in the murine model. Based on the pharmacological dose in 35 - to 84-day human trials $\left(0.5 \times 10^{11}\right.$ to $\left.1 \times 10^{11} \mathrm{CFU} / \mathrm{d}\right)(26,27)$, total extrapolated loads in mice can be about $1 \times 10^{10}$ to $3 \times 10^{10} \mathrm{CFU}$ with consideration of body weight and frequency. For safety in acute exposure, the actual treatment dose was reduced to $2 \times 10^{9} \mathrm{CFU}$, which corresponds to one-tenth of the total extrapolated levels. Mice were twice exposed via gavage to $10^{9} \mathrm{CFU} / 0.2 \mathrm{ml}$. After 2 rounds of bacterial inoculation in the gut, the recombinant bacteria $(\mathrm{EGF}-\mathrm{EcN})$ were detected in the stool by PCR analysis using primers specific to $\mathrm{EcN}$ (Figure 1D). The number of $\mathrm{EcN}$ (CFU) was estimated based on the relationship between the PCR threshold cycle and the apparent number of bacteria (28). On the 30th and 60th days after the initial 2 inoculations $\left(2 \times 10^{9} \mathrm{CFU}\right)$, bacterial colonization levels were decreased by a factor of $10^{4}$ (Figure 1D). Notably, the recombinant probiotic bacteria were detected even on the 60th day after gavage, 
A

$\frac{\text { IBD-Sleiman }(n=58)}{\text { IBD-Vemeire }(n=194)} \quad \frac{\text { IBD-Haberman }(n=322)}{\text { Colon only }}$
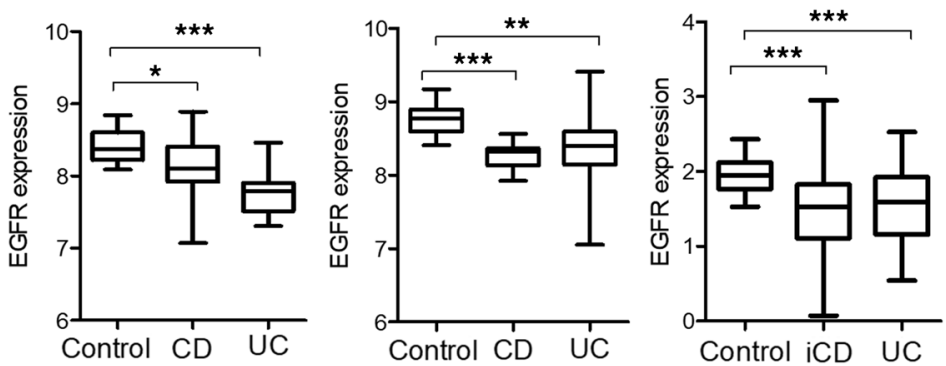

B

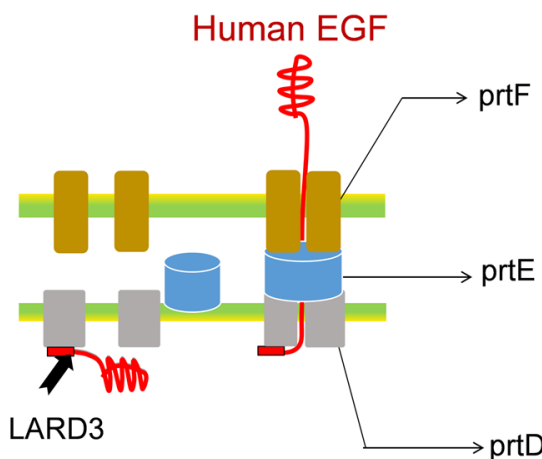

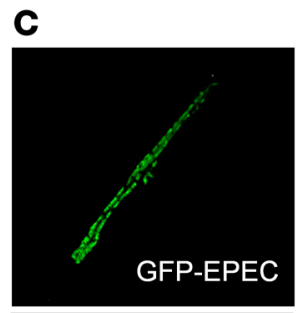

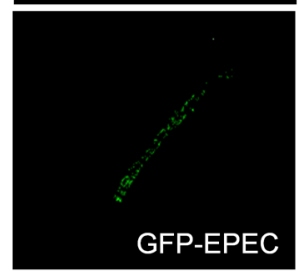

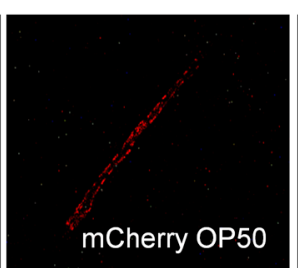

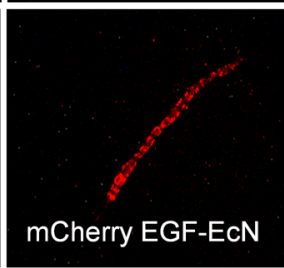

(100x magnification)
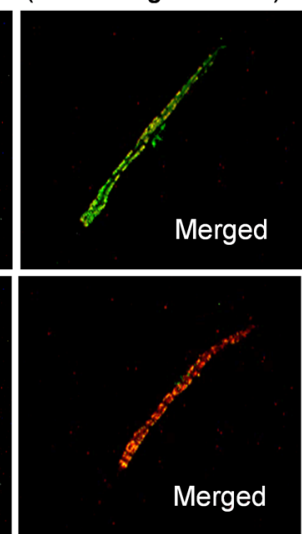

Nissle 1917 chromosome

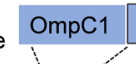

$\mathrm{OmpC}$

Crossing-over Recombination

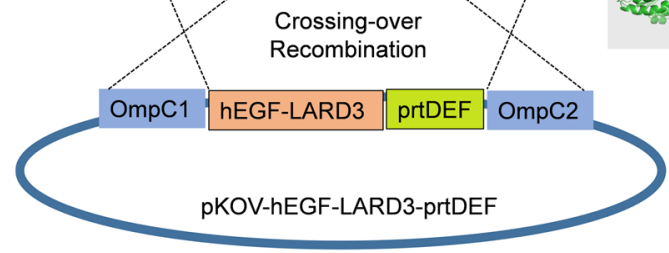

D

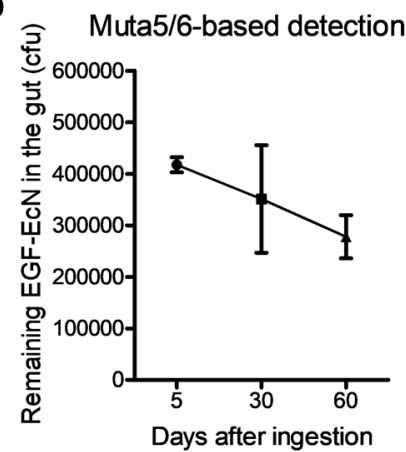

E

$$
\begin{array}{r}
\text { Fluorescence } \\
-4500 \\
-4260 \\
-3540 \\
-2820 \\
-2040 \\
-1320 \\
=600 \\
=\quad 420 \\
\\
\text { Intensity Unit }
\end{array}
$$

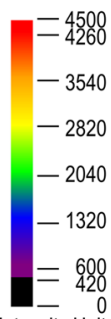

(Intensity/Min/Gain)

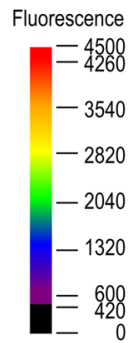

Intensity Unit

(Intensity/Min/Gain)

\section{$4^{\text {th }}$ day vehicle}

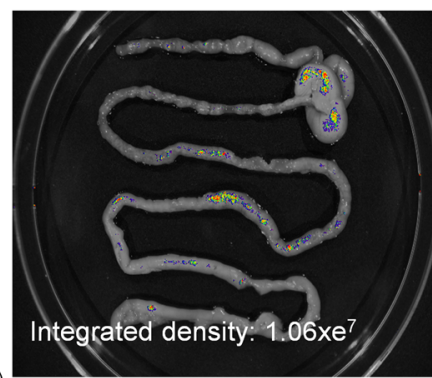

$29^{\text {th }}$ day vehicle

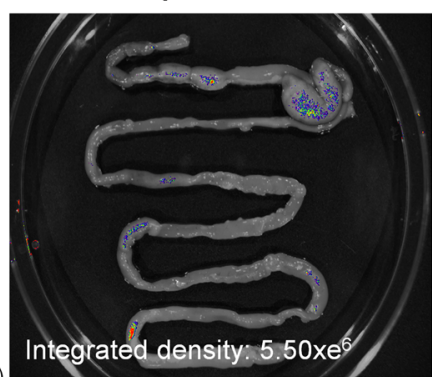

$4^{\text {th }}$ day EGF-EcN gavage
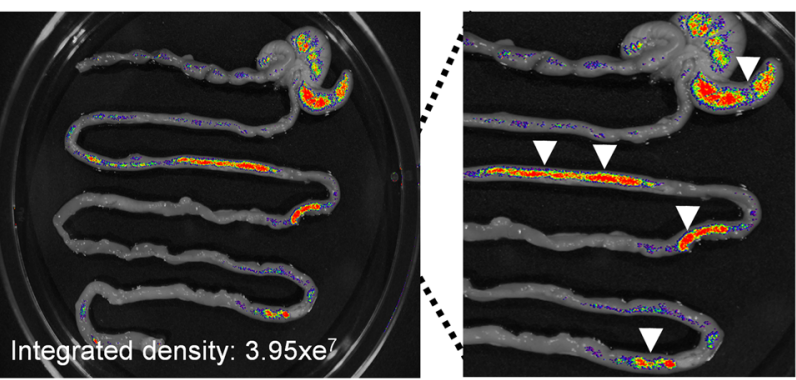

$29^{\text {th }}$ day EGF-EcN gavage

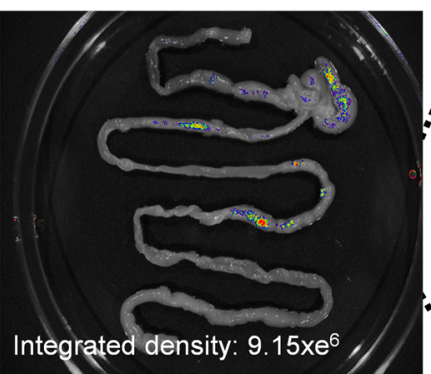

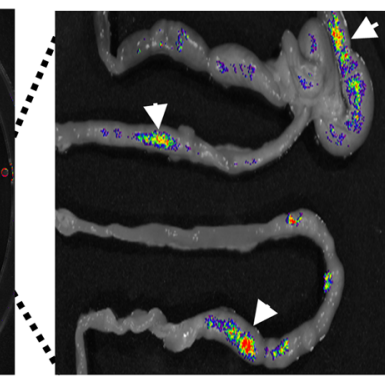


Figure 1. Construction and application of EGF-EcN in experimental gut models. (A) EGFR expression levels in the colon or ileum of patients with Crohn's disease (CD) or ulcerative colitis (UC). Three data sets (Sleiman's, Gene Expression Omnibus [GEO] ID GSE10616, $n=58$; Vemeire's, GEO ID GSE75214, $n=$ 194; and Haberman's GEO ID GSE57945, $n=322$ ) were compared. Haberman's samples were only from the ileum from patients with UC or ileocolonic CD (iCD). The asterisks in box-and-whisker plots (min to max) represent significant differences between 2 groups $\left({ }^{*} P<0.05,{ }^{* *} P<0.01\right.$, and ${ }^{* * *} P<0.001$ using 2-tailed, unpaired Student's $t$ test). The box plot depicts the minimum and maximum values (whiskers), the upper and lower quartiles, and the median. The length of the box represents the interquartile range. (B) The scheme for the bacterial secretion of LARD3-linked EGF protein through prtDEF. Genes for human mature EGF polypeptide linked to 1 fragment of LARD3 and prtDEF in pKOV vector were encoded in the bacterial chromosome by a crossing-over recombination at the OmpC region. Based on SWISS-MODEL (https://swissmodel.expasy.org/), expected binding modes in the bacterial membrane are depicted, based on SWISS-MODEL. (C) Colonization activity of E. coli OP50 and EGF-ECN in C. elegans intestine in the presence of mucoadherent enteropathogenic E. coli (EPEC). Gut colonization was assessed in wild-type (N2) C. elegans after 24 hours of preincubation with mCherry-labeled nonpathogenic E. coli (OP50 or EGF-EcN) in the presence of GFP-labeled EPEC (original magnification, $\times 100)$. The pictures are representative of 10 independent observations. (D) Stool samples from EGF-EcN-treated mice were collected at indicated times after gavage. Colonized bacteria levels were estimated based on PCR with EcN-specific primers for Muta5/6. (E) GFP-labeled EGF-EcN $\left(1 \times 10^{9} \mathrm{CFU} / 200 \mu \mathrm{L}\right)$ in the gut after gavage. The isolated intestines were observed on the 4 th and 29 th days after gavage. The colonizing bacteria were visualized in the intestine via an in vivo imaging system.

indicating long-term colonization with prolonged pharmacological actions. The recombinant EGF-EcN transformed with GFP encoding pGFP-UV vector was applied to mouse gut via gavage and visualized in the mouse intestinal mucosa. On the fourth day after gavage, the fluorescent recombinant probiotic bacteria were localized throughout the small intestine (Figure 1E). On the 29th day after gavage, some colonies with reduced signal were visualized in several parts of the small intestine.

Preventive or postinjury therapeutic action of EGF-EcN against colonic ulcers. To determine whether the EGF$\mathrm{EcN}$ is effective in ulceration models, EGF-EcN was administered to mice with colitis by dextran sodium sulfate (DSS). Mice were pretreated with EcN or EGF-EcN by oral gavage (in total, $2 \times 10^{9} \mathrm{CFU}$ ) and then treated with 3\% DSS for 5 days to induce colitis (Figure 2A). Because EcN has antibacterial activity (Figure 1C), it can affect the bacterial community in mice with colitis. Chemical-induced (DSS) colitis decreased the composition of phylum Firmicutes (Supplemental Figure 1B) and the Firmicutes to Bacteroidetes (F/B) ratio (Supplemental Figure 1C). However, treatment with EGF-EcN did not produce significant shifts in the bacterial community, at the phylum level, of mice with colitis. However, DSS-induced loss of body weight was markedly attenuated by probiotic treatment (Figure 2B). Notably, EGF-EcN had more preventive effects on ulceration-associated weight loss than the native EcN. Based on the extended observation of weight recovery after ulceration, mice inoculated with EGF-EcN showed the most rapid rebound weight gain. DSS-treated mice developed colitis, which was indicated by persistent diarrhea and shrinking of the colon, common symptoms of an inflamed intestine (Figure 2C). The presence of blood in mouse feces was detected at 5-6 days after the start of DSS treatment, whereas gross diarrhea was initially observed on day 4. Although shortening of the colon was marginally prevented by treatment with the vehicle probiotic bacteria, the colorectum preexposed to EGF-EcN maintained nearly normal histological appearance with no diarrhea or colorectal bleeding (Figure 2C), indirectly substantiating the protective effects of EGF-EcN against chemical-induced murine colitis. Next, pharmacological actions of EGF-EcN were addressed at the histopathological and cytological levels. The histopathological features in murine colitis are generally characterized by crypt distortion, inflammatory cell infiltration, ulceration, and destruction of the mucosal barrier. In agreement with these well-reported events, DSS treatment increased epithelial edema, inflammatory cell infiltration, mucosal ulceration, and crypt loss (Figure 2, D and E). In contrast, most DSS-linked histopathologic events were significantly suppressed by pretreatment with EGF-EcN, although the degree of reduction in intestinal disease scores was partial in EcN-pretreated mice (Figure 2E). In particular, the ulcer area was prominently reduced by treatment with EGF-EcN when compared with that in the colon epithelia of mice with DSS-induced colitis without any probiotic pretreatment (Figure 2F). Moreover, neutrophil infiltration in the mucosa as a readout of inflammation was visualized and quantified at original magnification $\times 400$ (Figure 2 G). EGF-EcN significantly attenuated mucosal inflammatory stimulation as well as the ulcerative injuries in the murine colitis model.

The preventive administration of EGF-EcN was effective against the chemical-induced colitis (Figure 2). In terms of therapeutic aspects, the recombinant probiotic agent was also assessed as a postinjury treatment. After colitis induction, animals were orally administered EGF-EcN every 3 days and the effectiveness was monitored (Supplemental Figure 2A). Although EGF-EcN treatment had marginal protective effects on DSS-induced weight loss during the early injury period, it partially enhanced body weight after the 12th day following DSS treatment (Supplemental Figure 2B). The postinjury treatment with EGF-EcN also enhanced colon length in gut-injured mice (Supplemental Figure 2, C and D). Although the preinjury 
A
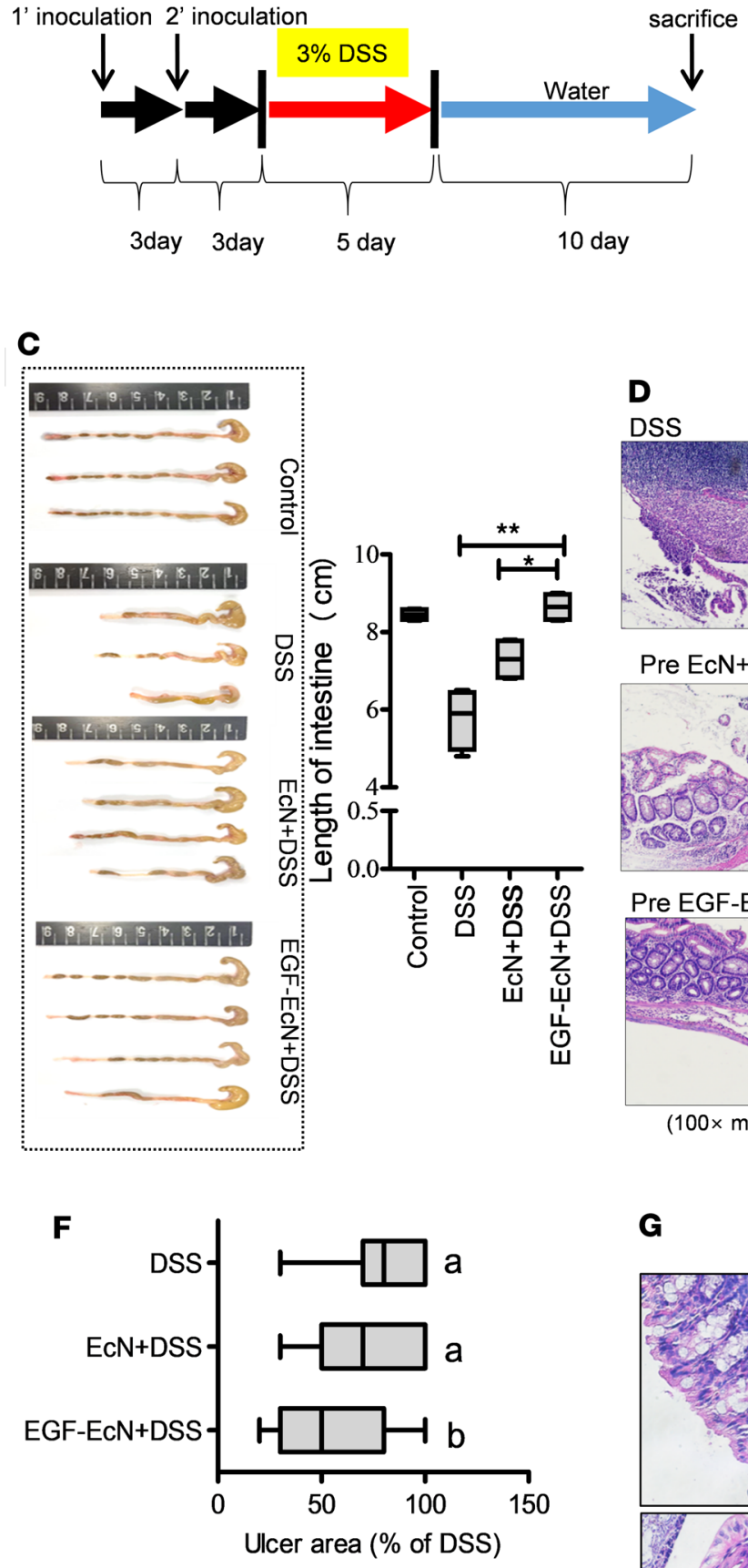

G
D

\section{oss}

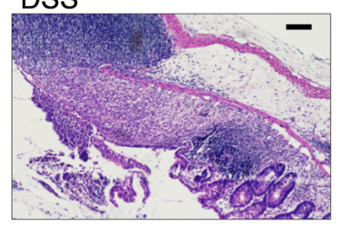

Pre $\mathrm{EcN}+$ post DSS

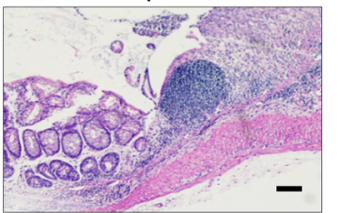

Pre EGF-EcN+DSS

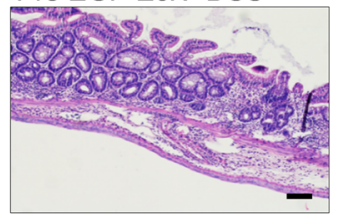

(100× magnification)

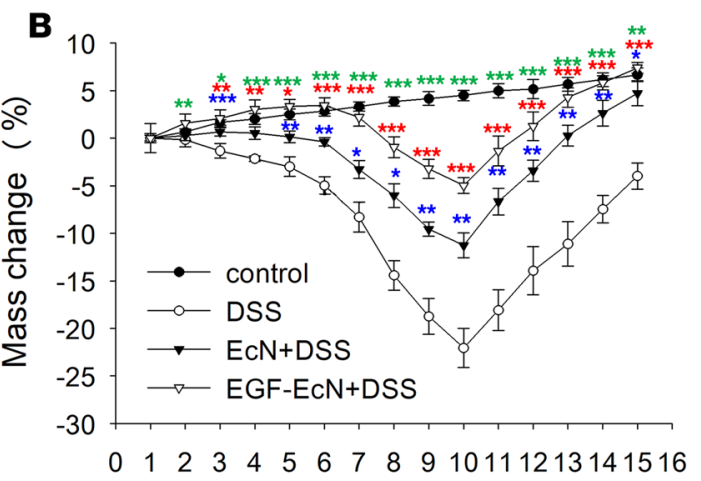

Day after DSS treatment
E

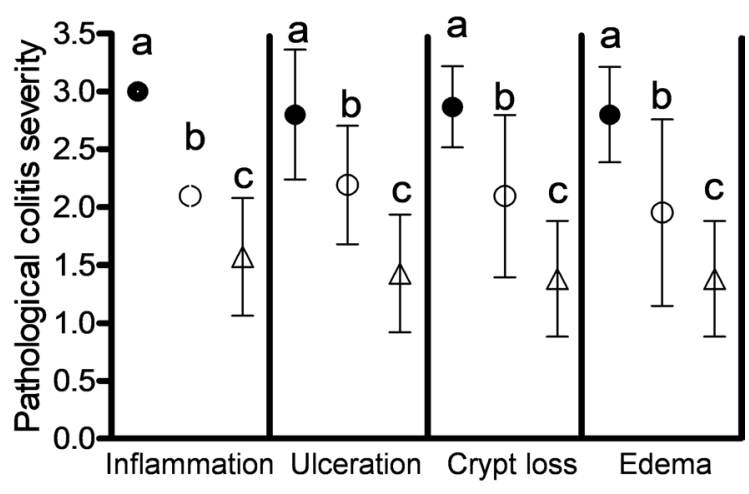

- DSS

O Pre EcN+post DSS

$\triangle$ Pre EGF-EcN+post DSS

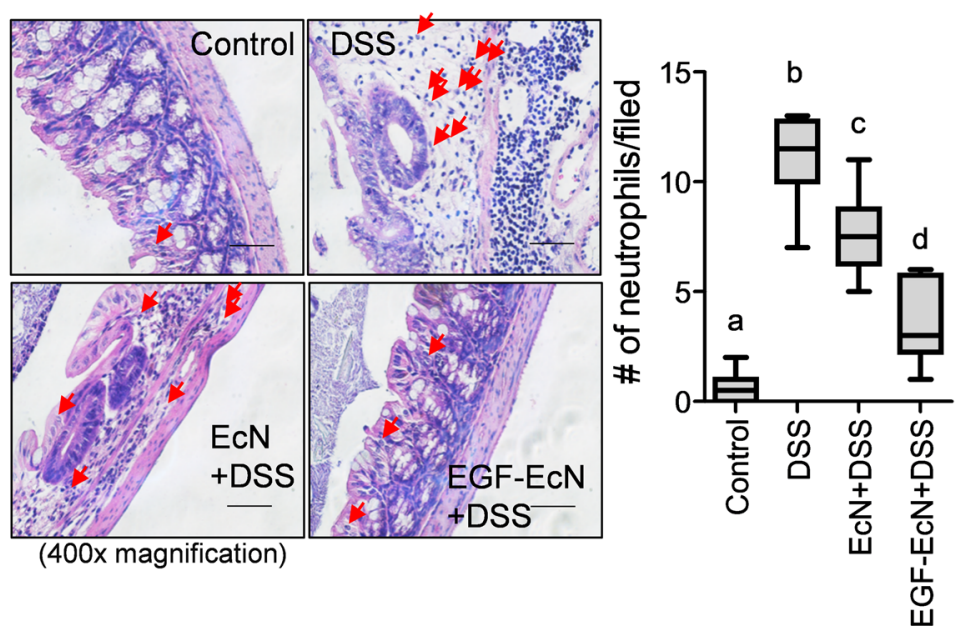

Figure 2. Actions of EGF-ECN in DSS-induced acute colitis. Schematic overview of the DSS-induced colitis model with EGF-EcN treatment. (A) Six-week-old female mice were pretreated twice with vehicle, EcN, or EGF-EcN over 7 days $(n=12-15)$. The mice were then exposed to $3 \%$ DSS for 5 days to induce colitis. (B) Mouse body weight was monitored for indicated times after DSS exposure. The asterisks in the graph represent significant differences from mass changes of the DSS treatment group at each time point $\left({ }^{*} P<0.05 ;{ }^{* *} P<0.01 ;{ }^{* *} P<0.001\right.$ using 2 -tailed, unpaired Student's $t$ test). (C) Changes in colon length were measured on the 10th day after DSS treatment (the left box) and quantitatively analyzed (the right graph). The asterisks in the box-and-whisker plot ( $\min$ to $\max$ ) represent significant differences between 2 groups ( ${ }^{*} P<0.05 ;{ }^{*} P<0.01$ using 2-tailed, unpaired Student's $t$ test). (D and $\mathbf{G})$ Representative hematoxylin and eosin staining in intestinal lesions demonstrated using microscopy. Original magnification, $\times 100$ (D), 400 (G). Scale bars: $100 \mu \mathrm{m}$. (E-C) Histopathological scores (E), levels of ulcer area (F), and neutrophil infiltration 
(G, red arrows) were compared between the DSS and EcN or EGF-EcN treatments. Results are shown as mean \pm SEM (E) or box-and-whisker plots (min to $\max$ ) (F and $\mathbf{G}$ ). Different letters represent a significant difference between groups in each parameter ( $P<0.05$ using 1 -way ANOVA with the Newman-Keuls post hoc test). The box plots depict the minimum and maximum values (whiskers), the upper and lower quartiles, and the median. The length of the box represents the interquartile range.

treatment was more notable in restoring body weight loss and colon shortening than the postinjury treatment, the colorectum exposed to EGF-EcN at the postinjury stage maintained nearly normal histological appearance (Supplemental Figure 2E). DSS-linked histopathological severity scores (epithelial edema, neutrophil infiltration, mucosal ulceration, and crypt loss) were significantly lowered by postinjury treatment with EGF-EcN (Supplemental Figure 2F). In particular, the ulcer area was reduced by postinjury treatment with EGF-EcN when compared with that in the colon epithelia of mice with DSS-induced colitis without any probiotic treatment (Supplemental Figure 2G). Taken together, both pre- and postinjury treatments of EGF-EcN were effective against DSS-induced ulcers in animals, suggesting they are a promising clinical intervention for human ulcerative intestinal diseases.

Effect of released EGF on cryptic proliferation in epithelial restitution. In terms of signaling transduction, EGFR phosphorylation in the intestinal epithelia was notably increased in mice preexposed to EGF$\mathrm{EcN}$ whereas EcN exposure alone marginally activated EGF-linked signals (Figure 3A). Furthermore, whole-colon lysates were analyzed for phosphorylation of downstream effector kinases, including ERK1/2 and AKT (Figure 3B). Phosphorylation of ERK1/2 and AKT were significantly enhanced by treatment with EGF-EcN in vivo compared with the levels in the other groups. Delivery of EGF from bacteria to the gut lumen was evaluated in the gut. In particular, LARD-tagged EGF protein was detectable around the crypt parts of the intestinal layer, where the luminal flow is slow or stagnant. EGFR activity was elevated around the region with high levels of EcN-secreted EGF, and the relative levels of phosphorylated EGFR (p-EGFR) were enhanced by treatment with the recombinant bacteria in the colonic mucosa (Figure 3C). In response to ulcerative injuries, restitution or regeneration of colonic epithelial cells from the crypt is crucial for maintaining the integrity of the intestinal barrier. Expression of Sox-9 as a representative biomarker of highly proliferating gut progenitor cells was measured in gut epithelia (Figure 4A). EGF-EcN significantly elevated levels of Sox-9 in the cryptic epithelial cells of mice exposed to ulcerative DSS, indicating the recovery of damaged epithelial layers by promoting cell proliferation from crypt progenitors. Moreover, highly proliferating cryptic parts of epithelial layers were observed by measuring the incorporation of DNA precursor BrdU into cells (Figure 4B). Of interest, EGF-EcN elevated the proliferation of cryptic epithelial cells in mice exposed to ulcerative DSS whereas $\mathrm{EcN}$ itself marginally enhanced the proliferation compared with the controls or with mice with DSS-induced colitis. The localization of BrdU-positive cells was unrestricted in the deeper parts of the crypt, but more cells with high proliferation activities moved upward (Figure 4B), indicating that EGF-EcN administration promotes the restitutive recovery of damaged epithelial layers by enhancing intestinal epithelial cell proliferation and upward migration from the crypt.

Effect of probiotic EGF on gut epithelial and mucosal barrier integrity. To address the biological actions of EGF in the gut of patients with ulcerative injuries, EGFR-associated correlations with key effect molecules were assessed by examining gene expression profiles of IBD-based data sets. In terms of gut barrier integrity, junctional molecules claudin-3 (CLDN3) and zonula occludens-1 (ZO-1 or TJP1), mucin (MUC2) production, and proliferating gut progenitor biomarkers for regeneration (LGR5 and SOX9) were found to be definitely associated with EGFR in patients with intestinal ulcerative injuries $(R=0.3-0.7)$ (Figure $5, \mathrm{~A}$ and $\mathrm{B}$ ). Of note, gut CLDN3, a crucial component of the epithelial tight junction, had the highest correlation with EGFR levels. Moreover, overall correlation coefficients in the colon-specific analysis were higher than the values for total gut samples from patients with IBD (Figure 5B). To monitor the EGF-linked biological actions, impacts of EGF-secreting bacteria were assessed by using immunohistochemical analysis of the insulted gut barrier of the experimental model. Because DSS-induced ulcerative injuries can be associated with gut barrier disruption or epithelial cell death, we assessed the related readouts by measuring the expression of junctional proteins (claudin-3 and TJP1) (Figure 5, C and D) and cytotoxicity-related genes (cleaved caspase-3) (Figure 5E). Exposure to ulcerative DSS downregulated expressions of claudin-3 and TJP1 in the gut epithelial layer, which were counteracted by treatment with EcN or EGF-EcN (Figure $5, \mathrm{C}$ and $\mathrm{D}$ ). Of note, EGF-EcN was prominent in attenuating the barrier disruption-related features. In terms of intestinal apoptosis, DSS treatment increased epithelial cleaved caspase-3 activation, but EGF$\mathrm{EcN}$-treated hosts showed reduced apoptosis responses in the gut barrier (Figure 5E). 
A
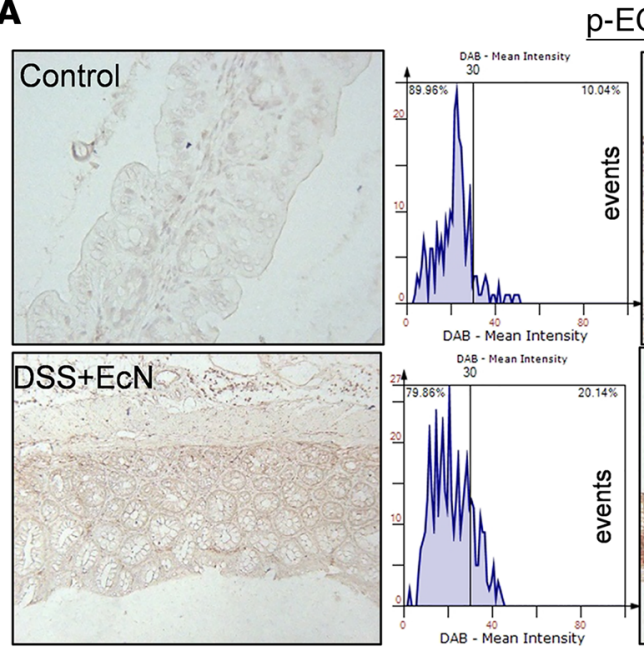

\section{EGFR}
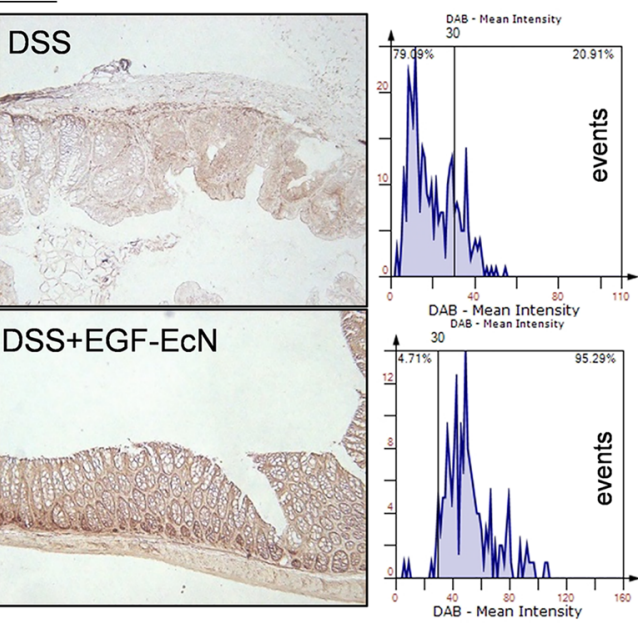

B

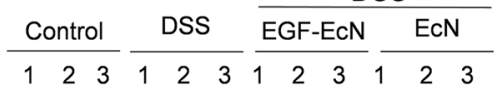

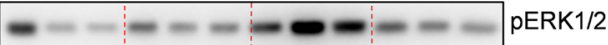

\begin{tabular}{llllll|llllllll}
1.0 & 0.6 & 0.5 & 0.9 & 0.7 & 0.8 & 2.1 & 3.9 & 3.1 & 0.9 & 0.9 & 0.8 & : relative intensity per ERK1/2
\end{tabular}

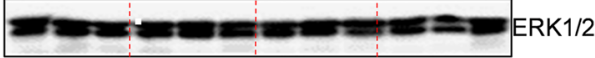

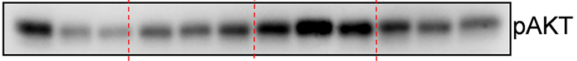

\begin{tabular}{llllll|lll|lll}
1.0 & 0.2 & 0.2 & 0.5 & 0.5 & 0.8 & 1.6 & 2.6 & 2.2 & 0.9 & 0.9 & 0.9
\end{tabular} : relative intensity per AKT

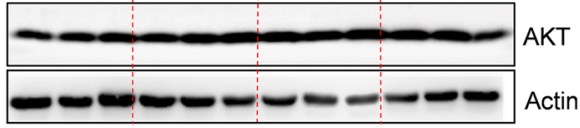

C

LARD-EGF

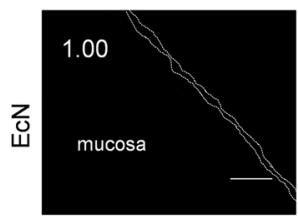

p-EGFR
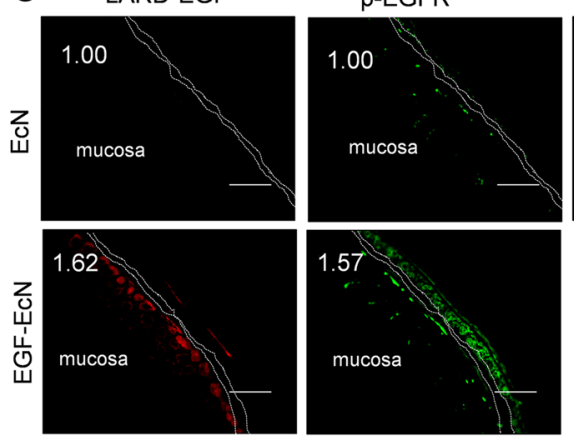

Merge

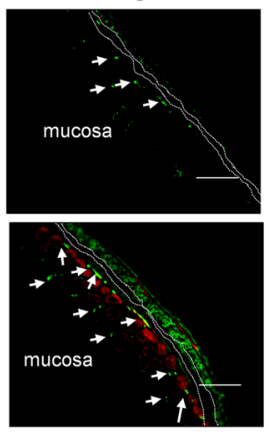

DAPI Merged

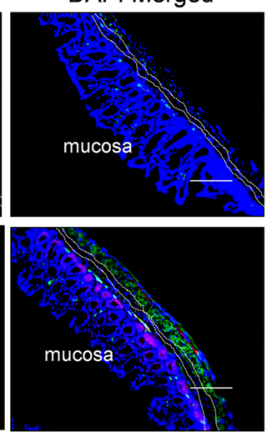

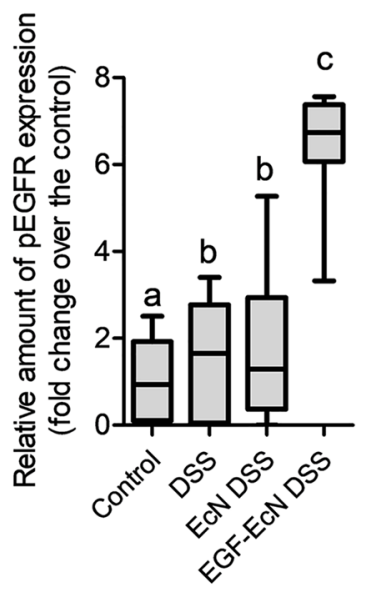

Figure 3. EGF-EcN-mediated actions in EGFR signaling in DSS-induced colitis. (A-C) Six-week-old female mice were pretreated twice with vehicle, EcN, or EGFEcN over 7 days $(n=12-15)$. The mice were then exposed to $3 \%$ DSS for 5 days to induce colitis. (A) Insulted colons were analyzed by IHC using the phosphorylated EGFR ( $p$-EGFR) antibody with hematoxylin counterstaining. A microscopy image at original magnification of $\times 200$ is shown at left. The relative density of $p$-EGFR was measured by using the HistoQuest tissue analysis software (each histogram of events with increasing diaminobenzidine (DAB) levels, described in the Methods section in detail). A quantitative comparison is shown in the right graphs. Scale bar: $100 \mu \mathrm{m}$. Results are shown as a box-and-whisker plot (min to max), and different letters represent a significant difference between groups ( $P<0.05$ using 1-way ANOVA with the Newman-Keuls post hoc test). (B) Each group of mouse colon lysates was subjected to Western blot analysis. The blots are representative of 3 independent experiments. (C) Eight-week-old female C57BL/ 6 mice were infected with $1 \times 10^{9} \mathrm{EcN}$ or EGF-EcN via oral gavage twice at 3-day intervals, and mouse gut was isolated on the third day after the second inoculation. LARDtagged EGF secreted from the EGF-EcN and activated EGFR were detected in the colonic mucosa. The white arrows indicate EGFR-Tyr1068 (green) in the mucosa, and the numbers in the upper left represent the relative levels of LARD-EGF or p-EGFR. Relative levels of p-EGFR in mucosa were compared in the box-and-whisker plot (min to max) (right), and the asterisks represent a significant difference between 2 groups ( ${ }^{* * *} P<0.001$ using 2 -tailed, unpaired Student's $t$ test).

In addition to the impacts on the junctional and adhesion molecules, the apical integrity of the gut barrier was assessed by measuring the distribution of apical F-actin (phalloidin staining). Closer observation of the murine intestinal epithelial barrier by staining of F-actin indicated that DSS treatment severely disrupted F-actin-based apical projections in the mouse gut epithelial lining, in which more F-actin molecules 
A

Sox-9

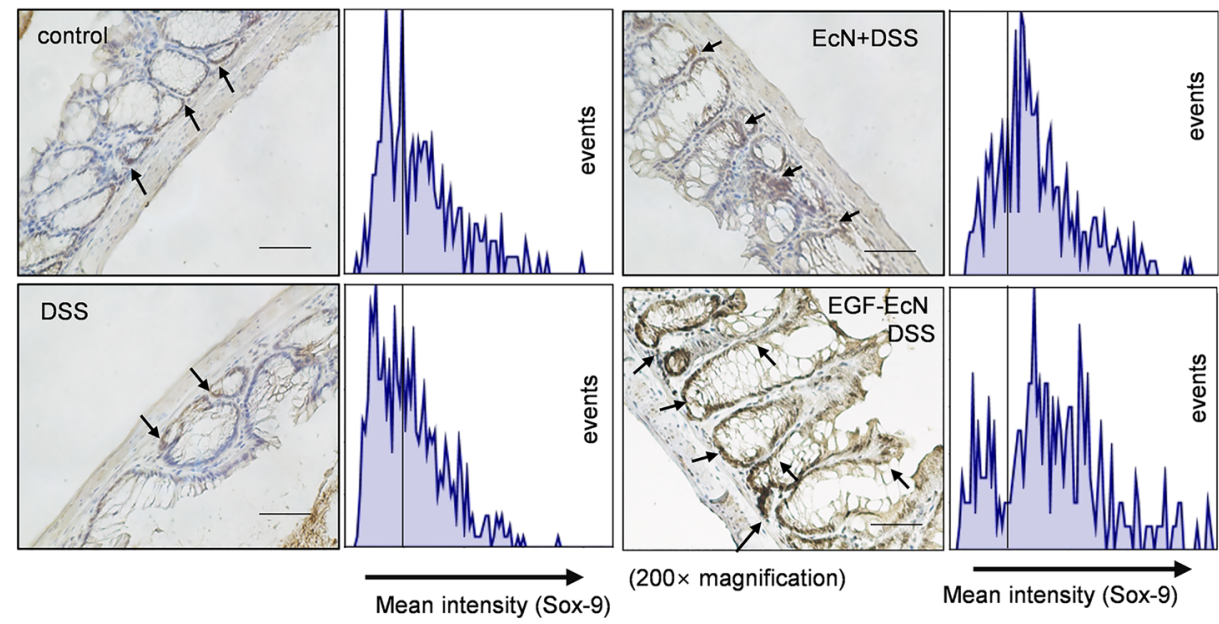

B
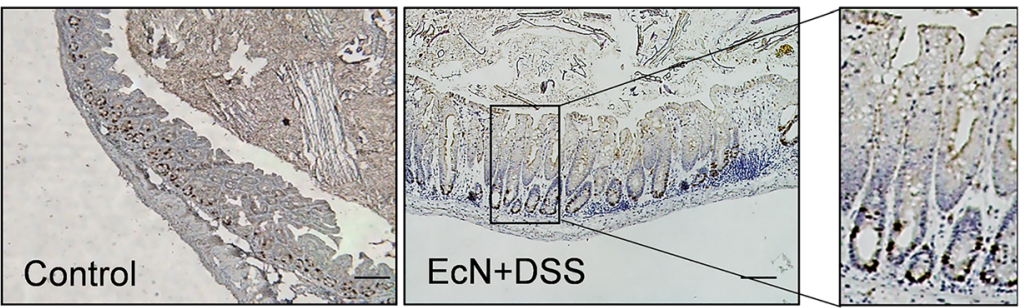

(100× magnification)
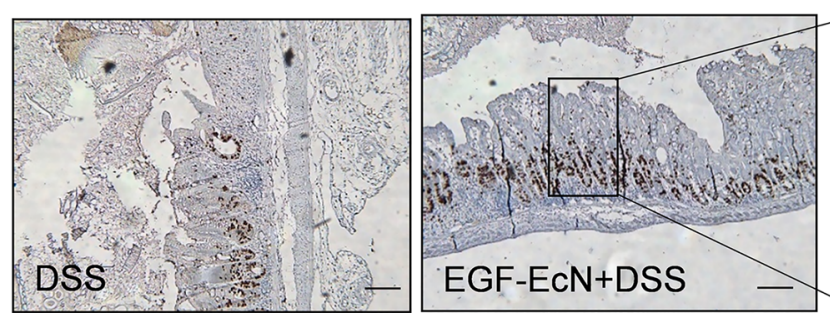

BrdU
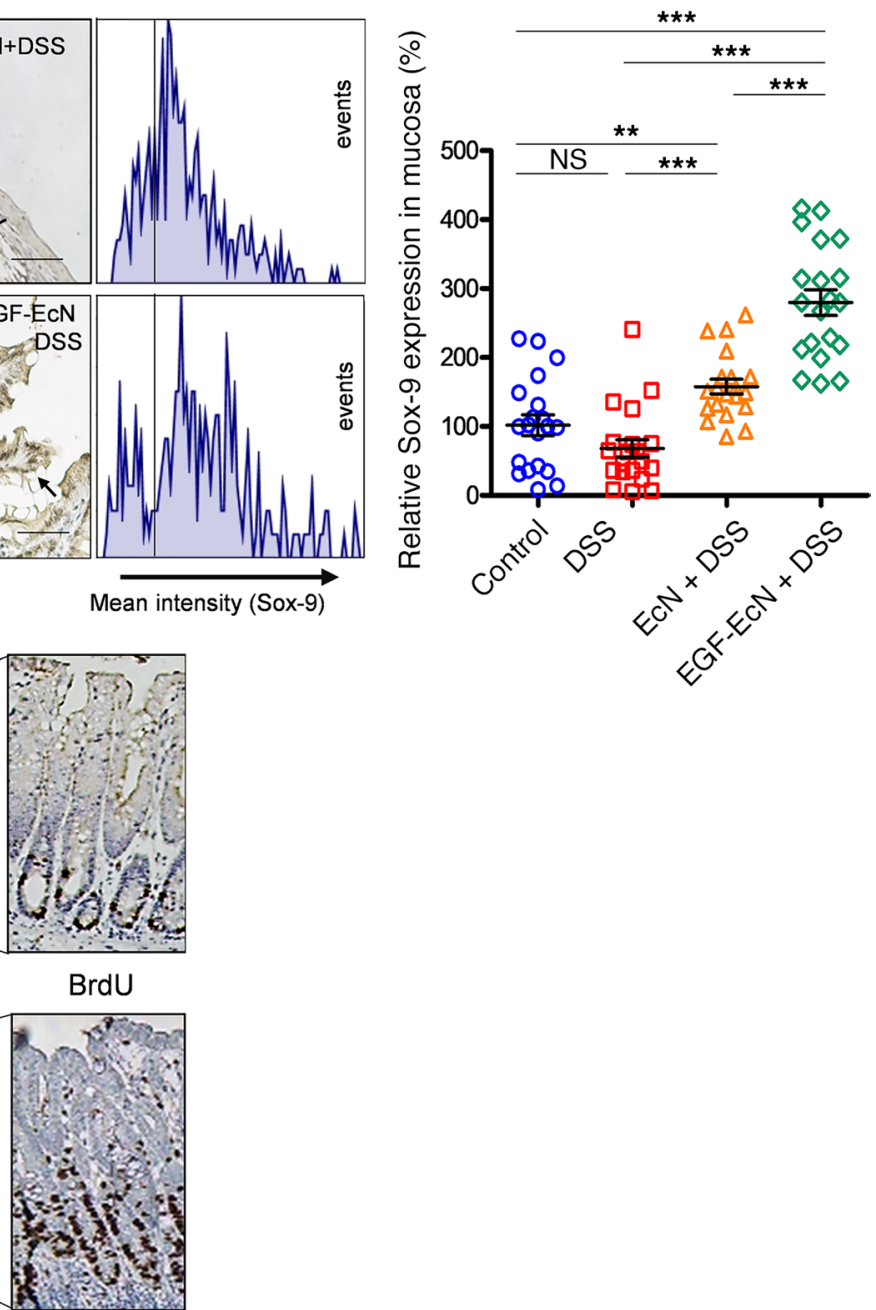

Figure 4. EGF-EcN-mediated cell proliferation in DSS-induced colitis. (A and B) Six-week-old female mice were pretreated twice with vehicle, EcN, or EGF-EcN over 7 days $(n=12-15)$. The mice were then exposed to $3 \%$ DSS for 5 days to induce colitis. (A) Sox-9 was detected and counterstained with hematoxylin (original magnification, $\times 200$ ). Scale bar: $100 \mu \mathrm{m}$. Each histogram represents events with increasing DAB levels. A quantitative comparison is shown in the right graph. The asterisks represent significant differences between the 2 groups $\left({ }^{* *} P<0.01\right.$; ${ }^{* *} P<0.001$; not significant [ns] using 2-tailed, unpaired Student's $t$ test). (B) Mouse colon tissues were stained for BrdU incorporation, and the nuclei were counterstained with hematoxylin (original magnification, $\times 100$; inset magnification, $\times 600$ ). Scale bar: $100 \mu \mathrm{m}$. The box plots depict the minimum and maximum values (whiskers), the upper and lower quartiles, and the median. The length of the box represents the interquartile range.

spread into the basolateral regions and even into the basement membrane (Figure 6A). However, treatment with EGF-EcN suppressed the disruption of the apical integrity in the intestinal epithelial layer. Along with the epithelial integrity improvement, mucosal layer integrity was improved by the recombinant probiotic bacteria because the organized secretion of mucus is facilitated by goblet cells, a secretory component of the epithelial lining of the gut mucosa. DSS treatment decreased the mucus secretion, which was notably restored by EGF-EcN treatment (Figure 6B). EcN also slightly improved the secretion of the mucus in insulted small intestine (Figure 6B), whereas it had marginal impacts on intestinal mucin secretion, Sox-9, and p-EGFR levels in the mice without ulcerative injuries (Supplemental Figure 3, A-C). Consequently, the improved secretion of mucus by EGF-EcN increased the thickness of the mucosal layer under the microbiota (Figure 6C). EGF-EcN-enhanced mucus secretion contributed to the improved integrity of the mucosal layer, which would limit luminal bacteria access. Because mice with chemical-induced colitis displayed disruption of epithelial and mucosal barriers, size-selective molecular translocation through the barriers can collapse. As another sign of a leaky gut barrier, large quantities of FITC-dextran were detected in the serum of mice with ulcerative injuries after oral administration. However, treatment with 


\section{A IBD-Sleiman $(n=58)$}
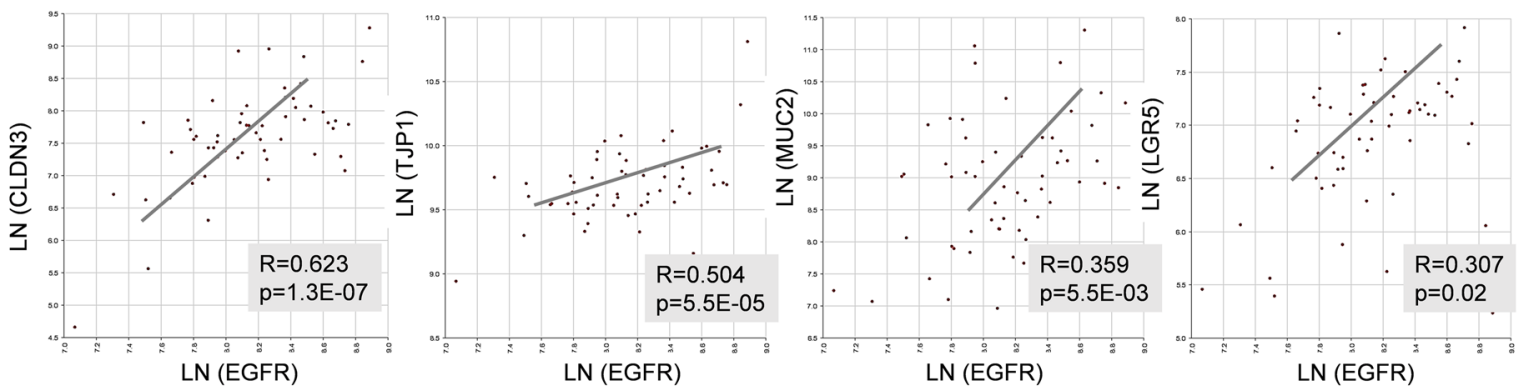

B $\quad \frac{\text { IBD-Vemeire }(\mathrm{n}=194)}{\text { Colon only }}$

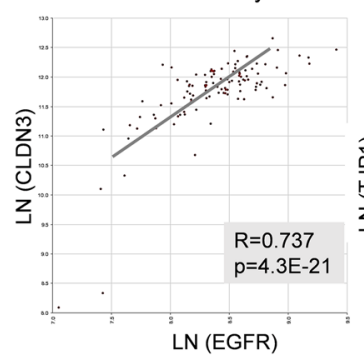

C

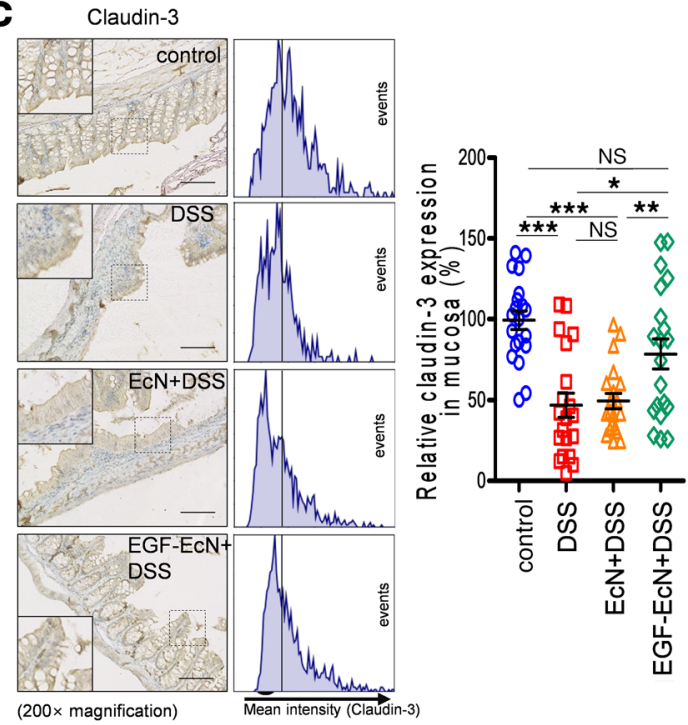

E

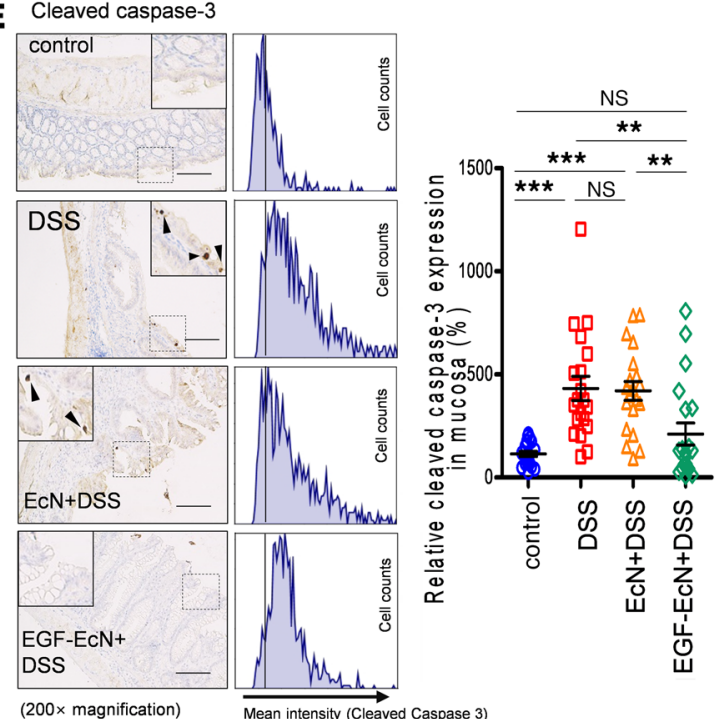

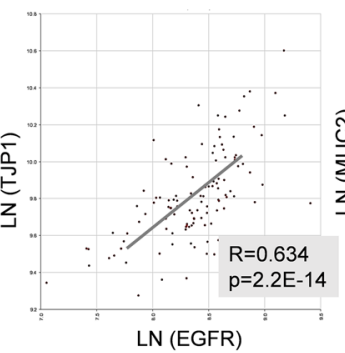

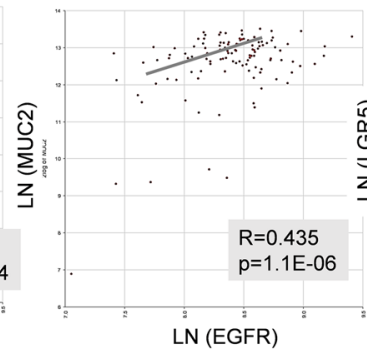

D
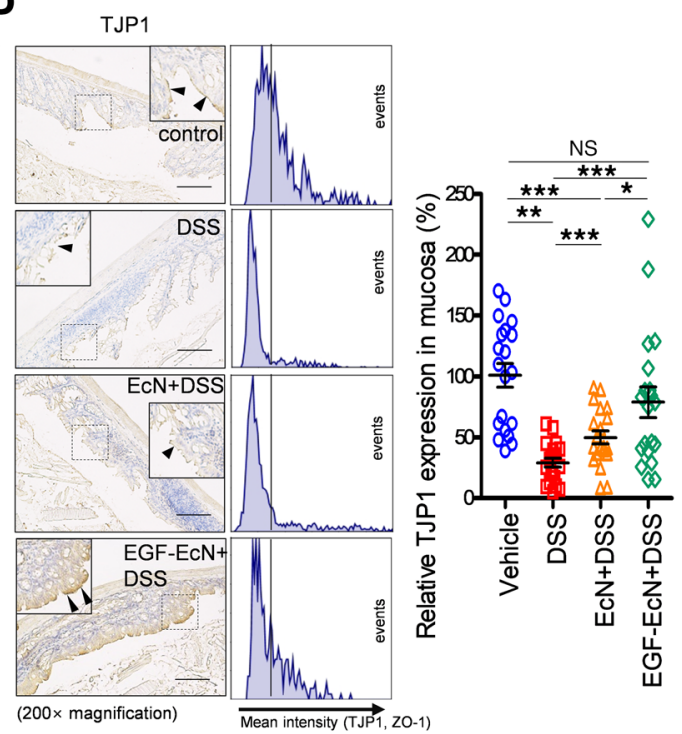

Figure 5. Links of EGFR expressions to gut barrier integrity. (A and B) The EGFR expressions were correlated with genes associated with gut barrier integrity (CLDN3, TJP1, MUC2, LCR5, and SOX9) in intestines of patients with IBD (Sleiman's, GEO ID GSE10616, $n=58$; Vemeire's, GEO ID GSE75214, $n=194$; and Haberman's GEO ID GSE57945, $n=194)$. Analysis of Vemeire's samples was performed with colonic mRNA (B). All values were based on Pearson's correlation analysis ( $R$, Pearson's correlation coefficient). (C-E) Six-week-old female mice were pretreated twice with vehicle, EcN, or EGF-EcN over 7 days $(n=12-15)$. The mice were then exposed to 3\% DSS for 5 days to induce colitis. Claudin-3 (C), TJP1 (D), and cleaved caspase-3 (E) were detected and counterstained with hematoxylin (original magnification, $\times 200$; inset magnification, $\times 800$ ). Scale bar: $100 \mu \mathrm{m}$. A quantitative comparison is shown in each right graph. The asterisks represent significant differences between 2 groups $\left({ }^{*} P<0.05\right.$; ${ }^{* *} P<0.01$; ${ }^{* *} P<0.001$; ns using 2-tailed, unpaired Student's $t$ test). 

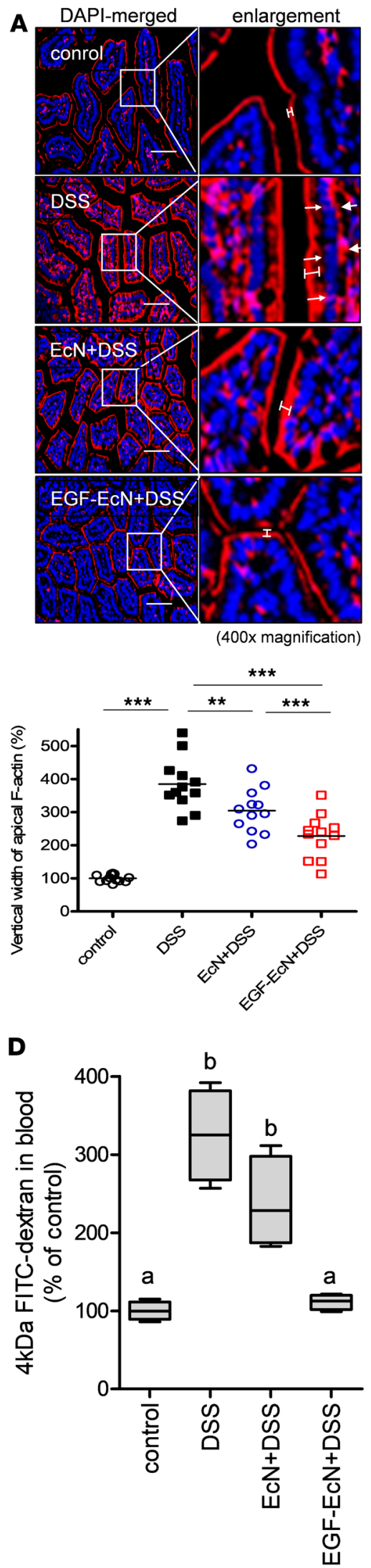

B
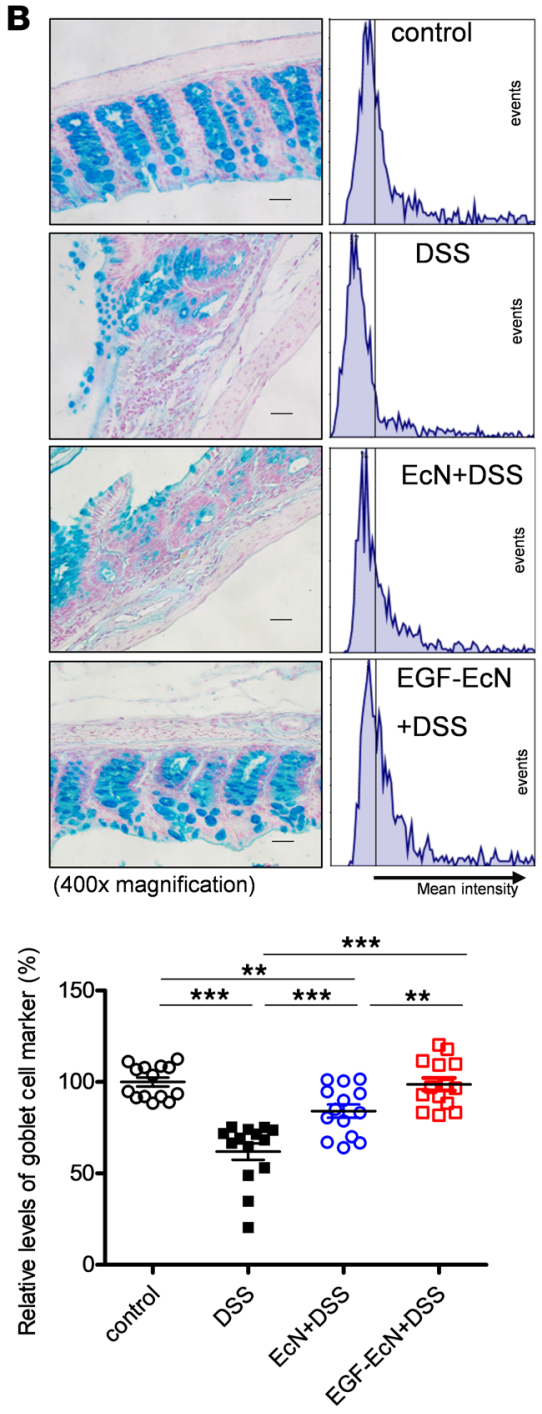

C

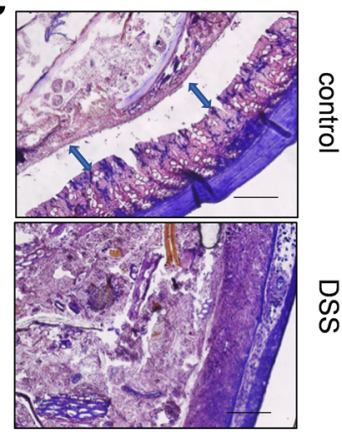

亨

œ
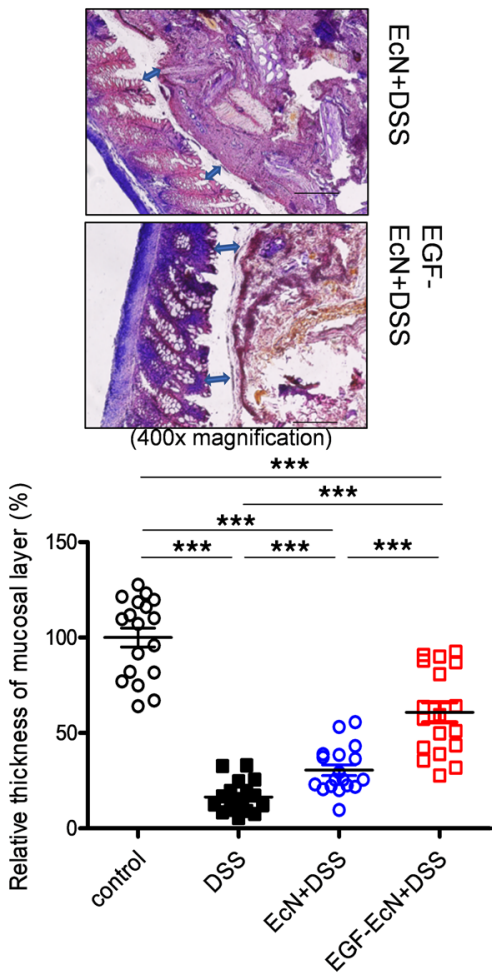

Figure 6. Effects of ECF-EcN on gut epithelial and mucosal barrier integrity in colonic ulcer. Sixweek-old female mice were pretreated twice with vehicle, EcN, or EGF-EcN over 7 days $(n=12-15)$. The mice were then exposed to 3\% DSS for 5 days to induce colitis. (A) Gut epithelia were stained with DAPI (blue) and phalloidin-TRITC (red) to stain nucleic acid and F-actin (original magnification, $\times 400$; inset magnification, $\times 8000$ ). Scale bar: $100 \mu \mathrm{m}$. White arrows indicate the spread of apical F-actin into the basement membrane. TRITC, tetramethylrhodamine. (B) Colons were isolated for mucin analysis. Secreted and intracellular mucins were stained with Alcian blue (blue colored, original magnification, $\times 400$. Scale bar: $100 \mu \mathrm{m}$ ). Each histogram represents events with increasing Alcian blue levels. A quantitative comparison is shown in the lower graph. (C) Gram staining to determine the accessibility of the total gut bacteria to the epithelial barrier (original magnification, $\times 400$. Scale bar: $100 \mu \mathrm{m})$. The asterisks represent significant differences between 2 groups (each lower graph in $\mathbf{A}, \mathbf{B}$, and $\mathbf{C})\left({ }^{* *} P<0.01 ;{ }^{* *} P<0.001\right.$ using 2-tailed, unpaired Student's $t$ test). (D) Intestinal permeability was evaluated at 3 hours after oral administration with FITC-conjugated dextran ( $150 \mathrm{mg} / \mathrm{kg}$ body weight). Results are shown as the box-and-whisker plot (min to max), and different letters represent significant differences between groups ( $P<0.05$ using 1-way ANOVA with the Newman-Keuls post hoc test). The box plots depict the minimum and maximum values (whiskers), the upper and lower quartiles, and the median. The length of the box represents the interquartile range. 
A

$1^{\prime}$ inoculation 2 ' inoculation

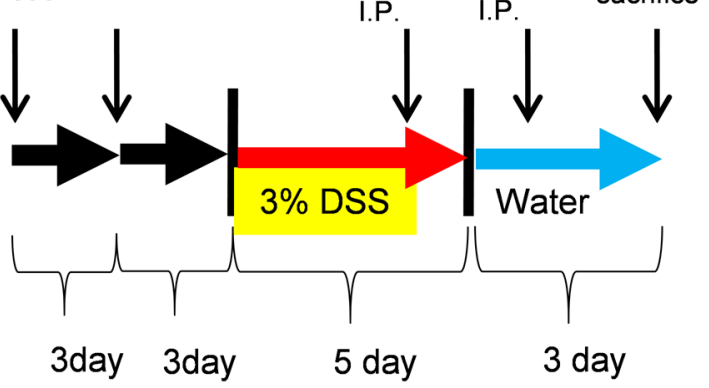

B

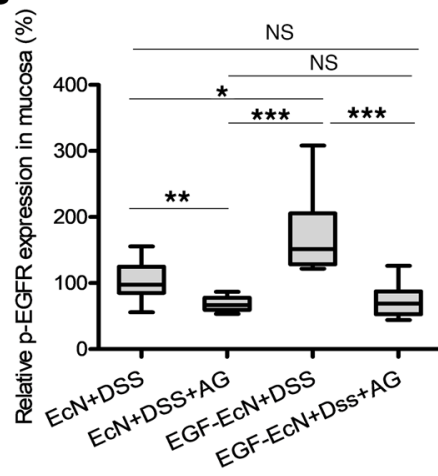

(200× magnification) anti-p-EGFR
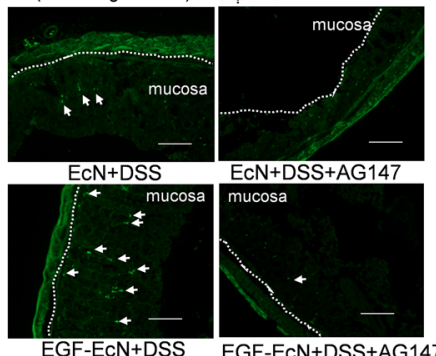

\section{C}

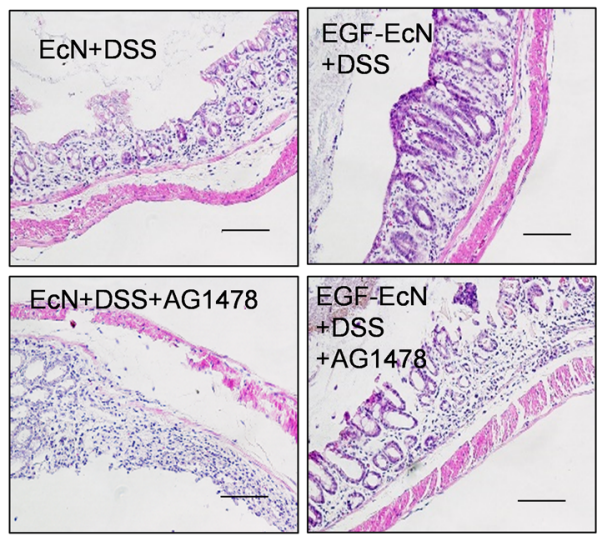

D
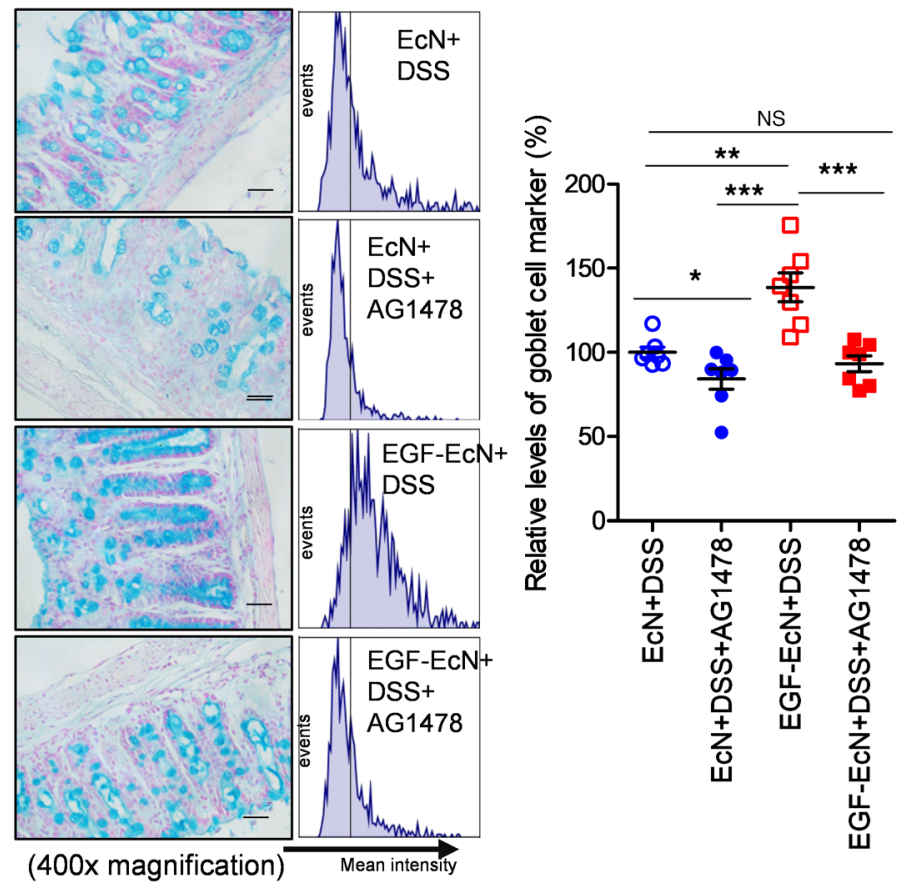

E

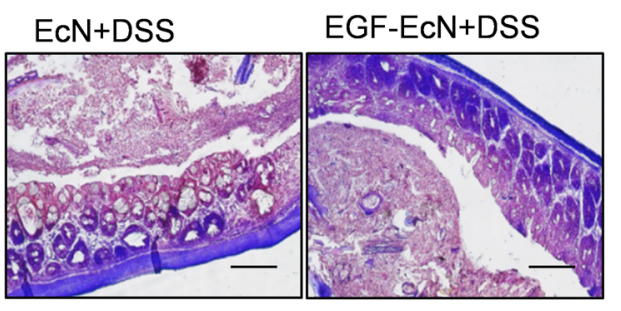

EcN+DSS+AG1478 EGF-EcN+DSS+AG1478
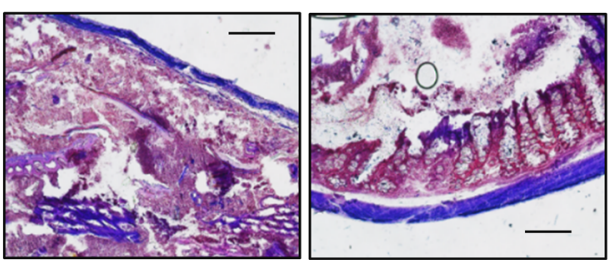

(400x magnification)

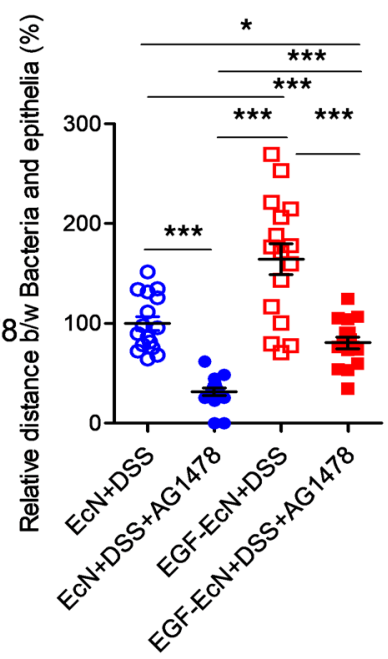

Figure 7. Involvement of EGFR signaling in recombinant bacteria-mediated protection. Eight-week-old female C57BL/6 mice $(n=8-15)$ were treated twice with $3 \%$ DSS after oral gavages with $1 \times 10^{9}$ EcN or EGF-EcN. Mice were then injected (intraperitoneally) with EGFR inhibitor (1 mg AG1478/mouse, Selleckchem) twice at 2-day intervals before and after the last day of DSS exposure. (A) Schematic outline of EGFR inhibition in the EcN or EGF-EcN treatment and DSS-induced colitis model. (B) Expression of p-EGFR in mucosa (green with the white arrow) was quantified (left box-and-whisker plot, 
min to max, based on fluorescence microscopy observations, shown at right) $\left({ }^{*} P<0.05\right.$; ${ }^{*} P<0.01 ;{ }^{* *} P<0.001$; ns using 2-tailed, unpaired Student's $t$ test). (C) Representative hematoxylin and eosin (H\&E) staining of the intestinal lesions as demonstrated by microscopy (original magnification, $\times 200$ ). Scale bar: $100 \mu \mathrm{m}$. (D) Colons were isolated for analysis of goblet cells and mucin production and were stained with Alcian blue (original magnification, $\times 400$. Scale bar: $100 \mu \mathrm{m})$. Each histogram represents events at an increasing Alcian blue level. A quantitative comparison is shown in the right graph $\left({ }^{*} P<\right.$ 0.05 ; ${ }^{* *} P<0.01$; ${ }^{* *} P<0.001$; ns using 2-tailed, unpaired Student's $t$ test). (E) Gram staining (original magnification, $\times 400$. Scale bar: $\left.100 \mu \mathrm{m}\right)$. The asterisks represent significant differences between 2 groups (the right graph) $\left({ }^{* *} P<0.01\right.$; ${ }^{* *} P<0.001$ using 2 -tailed, unpaired Student's $t$ test).

EGF-EcN almost completely suppressed FITC-dextran infiltration from the gut lumen (Figure 6D). To test whether the protective actions of EGF-EcN were accomplished through EGFR, we assessed the effects of EGFR inhibition on DSS-induced murine colitis (Figure 7A). A specific chemical inhibitor (AG1478) significantly attenuated EGFR activation by EGF-EcN in the gut mucosa (Figure 7B). Chemical inhibition of EGFR signaling also significantly attenuated $\mathrm{EcN}$ - or EGF-EcN-induced beneficial actions in the same DSS-induced colitis model (Figure 7C and Supplemental Figure 4, A and B). EGFR inhibition aggravated histology-based disease severity, manifesting as inflammation, crypt loss, and degree of ulcer area, in EcN- or EGF-EcN-treated mice under ulcerative stress (Supplemental Figure 4, A and B). Moreover, the recombinant bacteria-restored mucus secretion and thickening of the mucosal layer above the epithelial lining were also dependent on the EGFR-linked signaling pathway (Figure 7, D and E). Taken together, the engineered probiotic bacteria improved the colitis-associated pathological severity and gut barrier integrity in EGFR-dependent manners.

EGF-EcN attenuates ulcerative injuries in small intestine. Nonsteroidal antiinflammatory drugs (NSAIDs) are widely reported to cause severe ulcers in the upper gastrointestinal (GI) tract $(29,30)$. Moreover, with the recent increased use of sustained-release or enteric-coated formulations of NSAIDs, NSAID-induced damage to the distal GI tract, where the effects are difficult to monitor, has become more frequent (31). In the present study, an indomethacin-induced small intestinal injury model $(32,33)$ was used to assess the preventive action of EGF-EcN. As expected, the gross anatomy results demonstrated that lesions in the small intestine were increased by indomethacin treatment (Figure 8A). Of note, oral administration of indomethacin induced extensive spots of hemorrhage along the lining of the small intestine (Figure $8 \mathrm{~A}$ ). In contrast, the pathological events were attenuated in mice preexposed to EGF-EcN (Figure $8 \mathrm{~B})$. Histological examination showed defective villi, epithelial stratification, basal lamina degeneration, and infiltration of inflammatory cells into the epithelium of the small intestine of mice from a single indomethacin treatment. However, preexposure to EGF-EcN decreased scores for histopathological indexes related to small intestinal injury, such as inflammation, ulceration, crypt loss, and edema (Figure 8B). In detail, neutrophil infiltration as a biomarker of inflammation in the mucosa was visualized and quantified at original magnification $\times 400$ (Figure $8 \mathrm{C}$ ). Pretreatment with EGF-EcN significantly attenuated mucosal inflammatory stimulation as well as the ulcerative injuries in the NSAID-induced intestinal injury model. Moreover, ulcer area was prominently reduced by pretreatment with EGF-EcN when compared with that in the small intestinal epithelia of mice exposed only to indomethacin without any probiotic pretreatment (Figure 8D). In contrast with the preventive actions of EGF-EcN against NSAID-induced ulcerative injuries in the small intestine, the postinjury treatment with EGF-EcN was ineffective in mitigating the acute hemorrhagic toxicity in the small intestine (Supplemental Figure 5A). Histology-based disease severity, manifested as inflammation, ulceration, crypt loss, and edema, were not attenuated by the postinjury treatment of EGF-EcN (Supplemental Figure 5B). Moreover, quantitative analysis of ulcer area (Supplemental Figure 5C) confirmed the ineffectiveness of postinjury application of EGF-EcN against NSAID-induced intestinal injury.

In response to ulcerative insult, the proliferation of cryptic progenitor cells is expected to increase to compensate for the loss of the epithelial barrier, as shown in the DSS-induced colitis model. When the proliferation of progenitor cells was monitored by a tissue BrdU incorporation assay, indomethacin exposure also increased the number of highly proliferating epithelial cells per villus, and a greater increase was observed in response to pretreatment with EGF-EcN (Figure 9A). Moreover, the localization of BrdU-positive cells was not restricted in the lower parts of the villus, and more cells with high proliferation activities moved upward (Figure 9B), indicating that EGF-EcN administration promotes the restitutive recovery of damaged epithelial layers by enhancing cellular proliferation and migration from crypt parts of the small intestine. As a biochemical response to EGF, murine pretreatment with EGF-EcN also enhanced EGFR phosphorylation in the small intestinal epithelia, as shown by the immunohistochemical results (Figure 9C). As a growth 
A

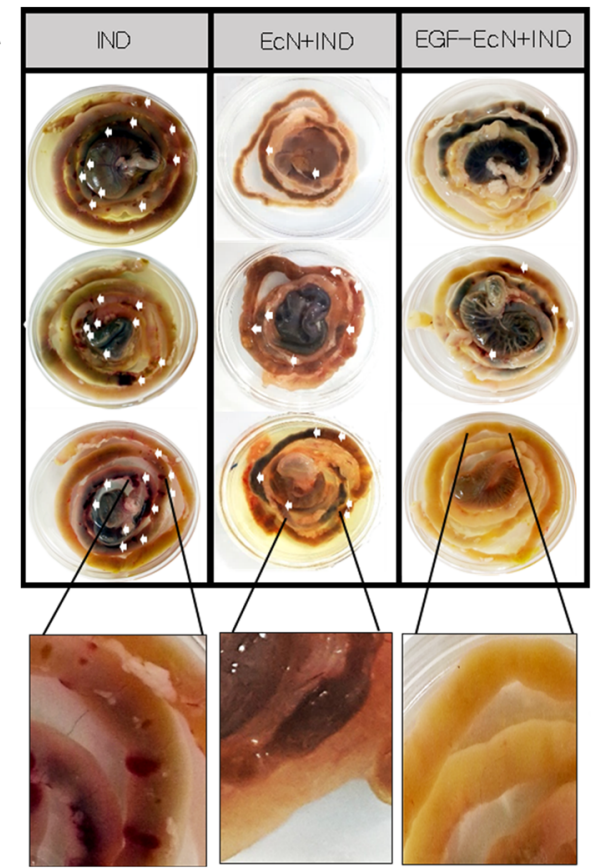

B
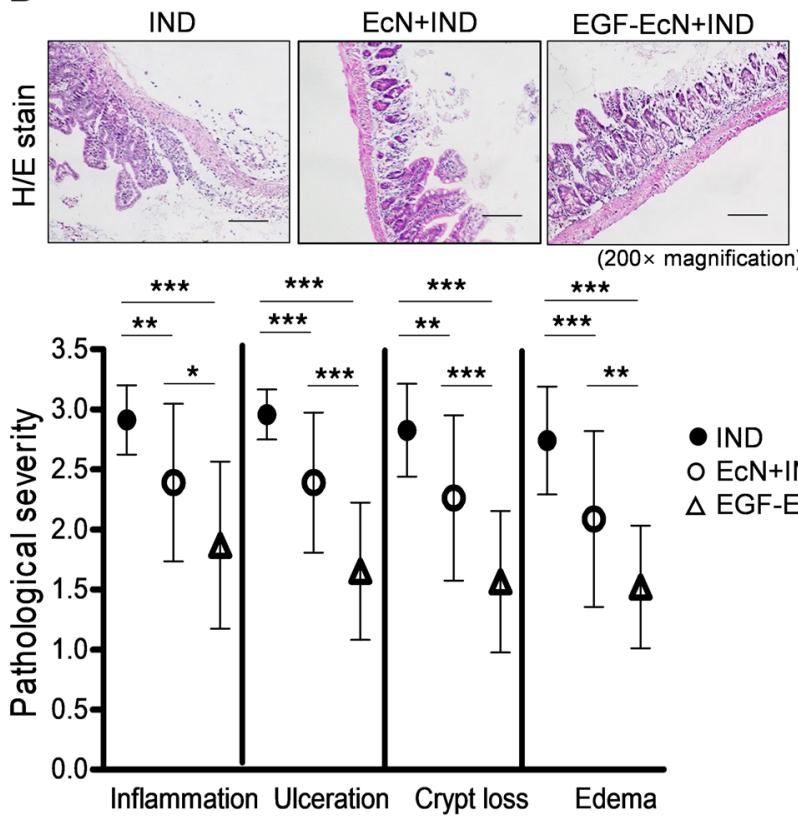

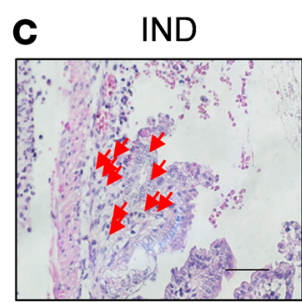

$\mathrm{EcN}+\mathrm{IND}$

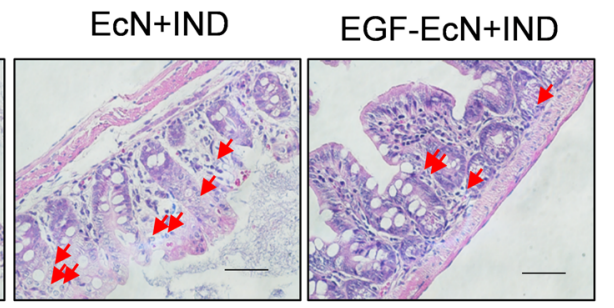

(400x magnification)
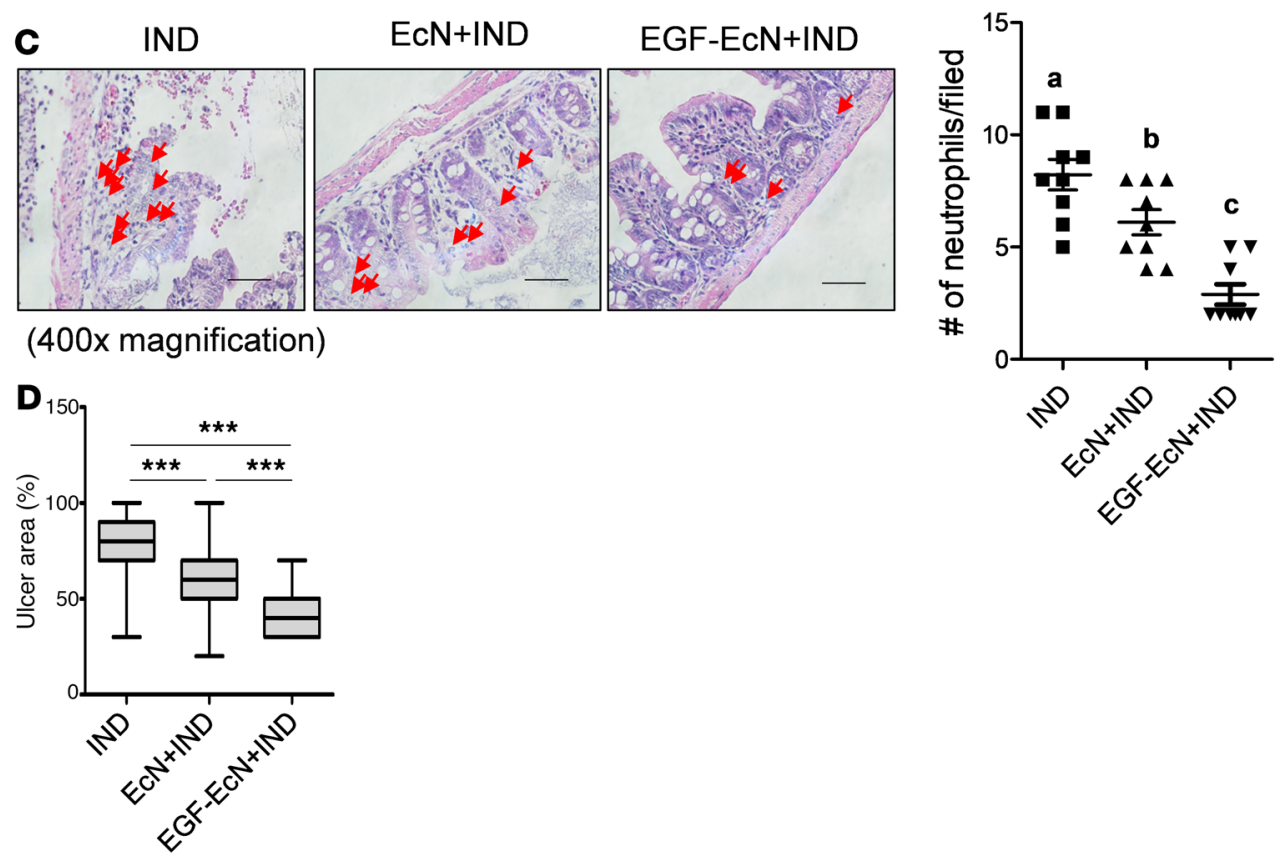

Figure 8. Effects of EGF-EcN on NSAID-induced ulcerative injuries in the small intestine. Ten-week-old male mice were pretreated twice with vehicle, EcN, and EGF-EcN over 7 days $(n=12)$. The mice were then treated with $30 \mathrm{mg} / \mathrm{kg}$ of indomethacin via gavage. (A) At 24 hours after indomethacin treatment, tissues were observed in "Swiss rolls" of small intestines and analyzed using a stereoscopic microscope. The white arrows indicate hemorrhages along the line of intestines. Inset magnification, x18. (B-D) At 24 hours after indomethacin treatment, tissues were assessed for H\&E staining patterns (B, upper), pathological severity score (B, lower), neutrophil infiltration (C, the representative images and the quantitation in the right graph), and ulcer area (D). Results in B are shown as mean \pm SEM with asterisks representing significant differences between 2 groups ( $P<0.05$; ${ }^{*} P<0.01 ;{ }^{* *} P<0.001$ using 2-tailed, unpaired Student's $t$ test). Scale bars: $100 \mu \mathrm{m}$. Different letters (C) represent significant differences between groups ( $P<0.05$ using 1-way ANOVA with the Newman-Keuls post hoc test). The asterisks in the box-and-whisker plot (min to max, D) represent significant differences between 2 groups $\left({ }^{*} P<0.05\right.$; ${ }^{*} P<0.01 ;{ }^{* *} P<0.001$ using 2-tailed, unpaired Student's $t$ test). The box plots depict the minimum and maximum values (whiskers), the upper and lower quartiles, and the median. The length of the box represents the interquartile range. 
A
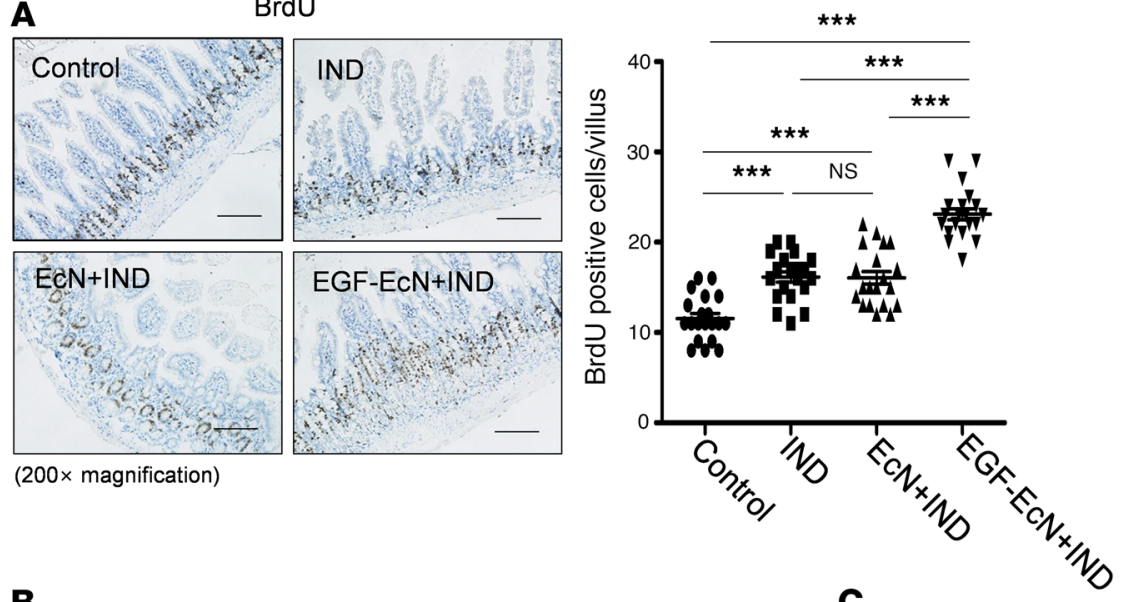

B

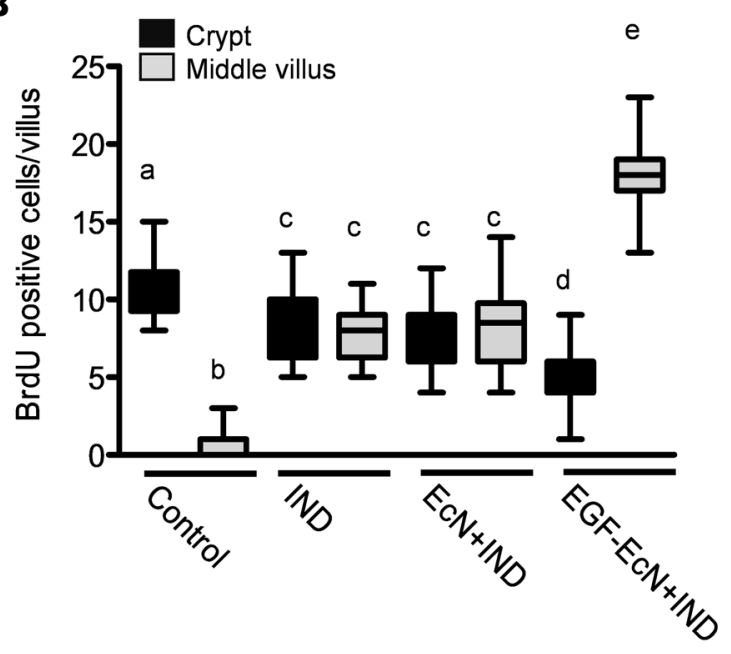

D Sox-9
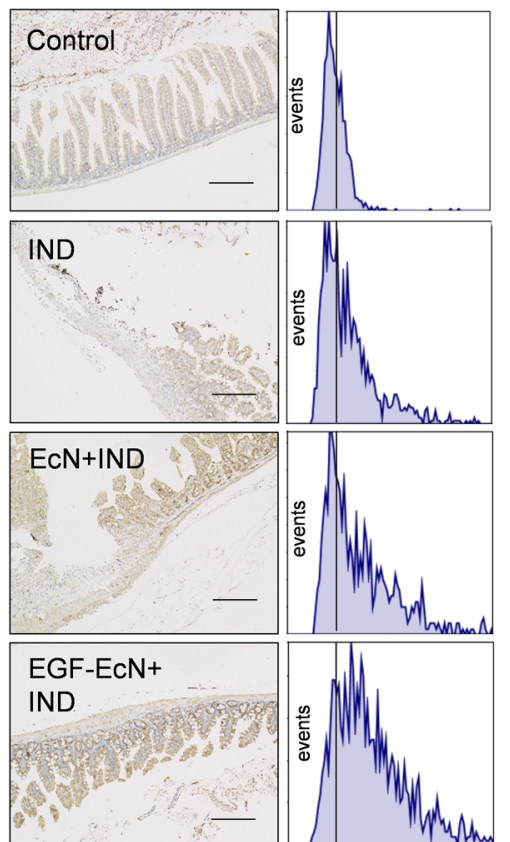

(200× magnification)

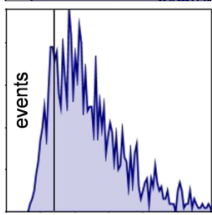

$\overrightarrow{\text { Mean intensity (Sox-9) }}$
C
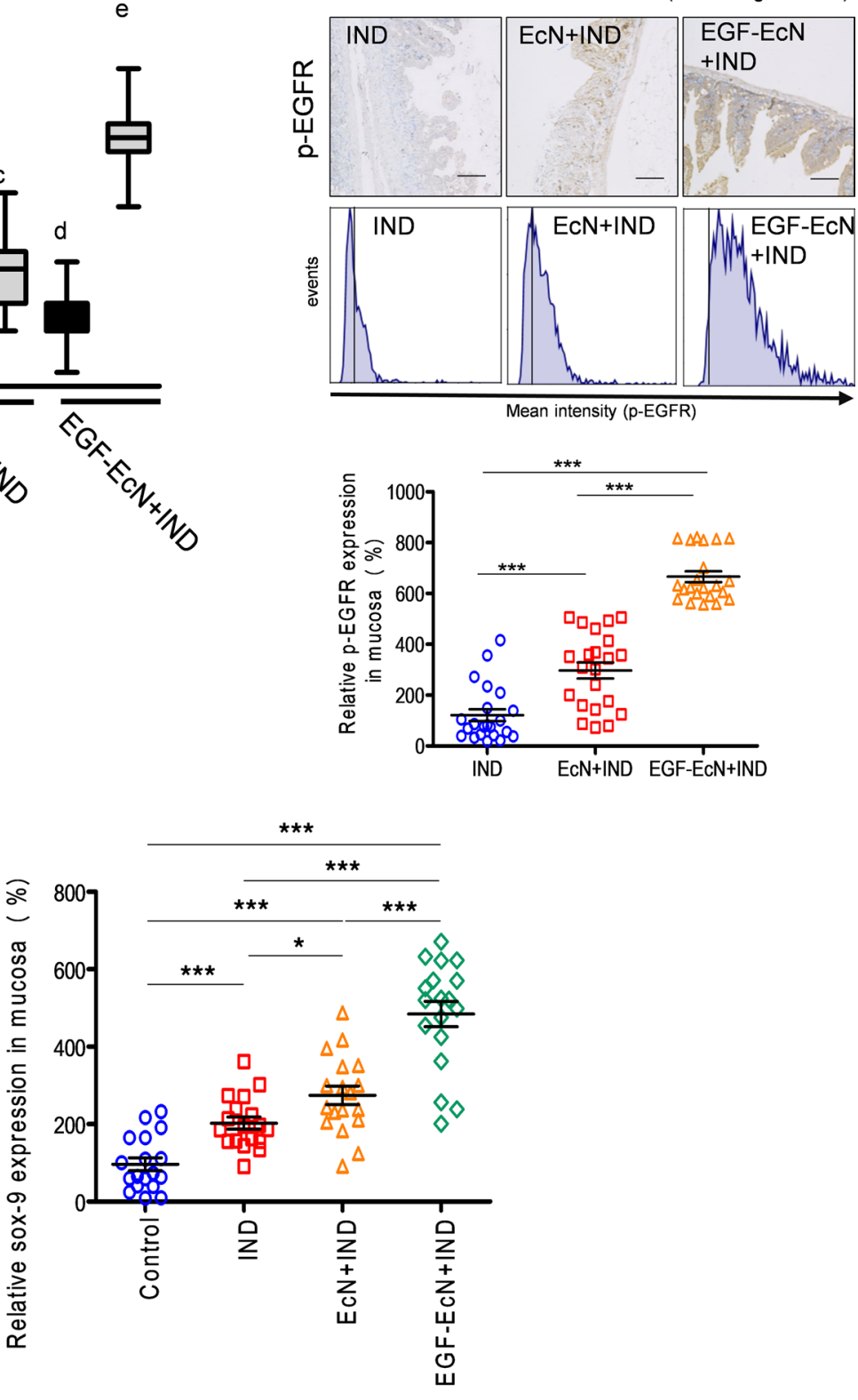
Figure 9. EGF-EcN-mediated actions in cell proliferation in NSAID-induced ulcer. Ten-week-old male mice were pretreated twice with vehicle, EcN, and EGF-EcN over 7 days $(n=12)$. The mice were then treated with $30 \mathrm{mg} / \mathrm{kg}$ of indomethacin via gavage. (A) Mouse small intestinal tissues were stained for BrdU incorporation while the nuclei were counterstained with hematoxylin (original magnification, $\times 200$ ). Scale bar: $100 \mu \mathrm{m}$. The BrdU-positive cells per villus were counted and the counts compared (***P 0.001 and ns using 2-tailed, unpaired Student's $t$ test). (B) The vertical intestinal villus was divided into the crypt part and the middle villus part. BrdU-positive cells in each part were counted per villus. Results are shown as the box-and-whisker plot (min to max), and different letters represent significant differences between groups $(P<0.05$ using 1 -way ANOVA with the Newman-Keuls post hoc test). The box plots depict the minimum and maximum values (whiskers), the upper and lower quartiles, and the median. The length of the box represents the interquartile range. (C) IHC staining using anti-p-EGFR antibody was assessed under the microscope (original magnification, $\times 200$ ). Scale bar: $100 \mu \mathrm{m}$. (D) Mouse small intestinal tissue was stained to detect Sox-9 protein and counterstained with hematoxylin. Each histogram represents events at an increasing DAB level. Scale bar: $100 \mu \mathrm{m}$. A quantitative comparison is shown in the graphs (C and $\mathbf{D},{ }^{*} P<0.05$; ${ }^{*} P<0.01 ;{ }^{* *} P<0.001$ using 2-tailed, unpaired Student's $t$ test).

factor response, expression of Sox-9 as a representative biomarker of proliferating gut progenitor cells in the cryptic area was also enhanced by colonization of EGF-EcN in indomethacin-induced intestinal ulcer models (Figure 9D). Taken together, pretreatment with the recombinant probiotic EGF-EcN promoted the intestinal wound healing process by enhanced proliferation of gut epithelial progenitor cells in the murine small intestinal ulcer model, indicating a promising preclinical pathway toward ultimate human uses.

EGF-EcN mitigates colitis-associated cancer in a mouse model. Although EcN-EGF was an effective regulator of inflammatory and ulcerative intestinal diseases, EGF could be detrimental because of its growth-promoting action for tumor cells. On the basis of this concern, EGF-EcN was assessed for its chronic effects on malignant diseases, such as inflammation-associated cancers. After a mutagenic hit by azoxymethane (AOM), 3 DSS-induced insults caused severe inflammation and subsequently increased colorectal tumors in the mouse model. EGF-EcN or EcN $\left(2 \times 10^{9} \mathrm{CFU}\right)$ was administered after each of the 3 DSS insults, and symptoms of the treated mice were monitored (Figure 10A). The remaining number of EcN (CFU) in the gut was estimated based on the relationship between the PCR threshold cycle and the apparent number of bacteria (28). On the 85th day after sequential inoculations (in total, $3 \times 2 \times 10^{9} \mathrm{CFU}$ ) after each round of chemical-induced ulcerative injury, the bacterial colonization levels were decreased by a factor of 3500 (Figure 10B). EGF-EcN treatment did not exacerbate the body weight fluctuation but slightly enhanced weight restoration after the final exposure to DSS while EcN treatment did not improve this event efficiently (Figure 10C). Although the recombinant probiotic EGF-EcN treatment marginally improved AOM/ DSS-induced colon shortening, it attenuated severe hemorrhoid protrusion by AOM/DSS treatment (Figure 10D). Furthermore, the histopathological observation of the gut demonstrated that EGF-EcN treatment significantly reduced the number of colon tumor masses (Figure 10, E and F) and tumor area (Figure 10G). Additional histological quantitation showed that the frequencies of the large tumors $\left(>0.9 \mathrm{~mm}^{2}\right)$ in mice treated with EGF-EcN was only $30 \%$ of the group without the probiotic exposure (Figure 11A). Moreover, the amount of high-grade dysplasia was also decreased by the recombinant probiotic bacteria (Figure 11B). Along with the effects of the recombinant probiotics on adenoma, the fibrosis-related signs were also assessed in the tumor mass. AOM/DSS exposure increased collagen deposition in both the mucosa and submucosa layers of the colon, which was attenuated by EGF-EcN treatment (Figure 11C). As another biomarker of fibrosis, $\alpha$-SMA expression in the tumor mass was also lowered by treatment with EGF-EcN (Figure 11D).

Although EGF-EcN definitely functioned in normal and malignant colonic tissues by activating EGFR (Figure 12A), the numbers and sizes of the epithelial tumors in the mucosa decreased in response to EGF$\mathrm{EcN}$ (Figure 10, E-G). In addition, the increase in tumor mass was measured by staining Ki-67, a representative biomarker of the proliferative activities. EGF-EcN treatment significantly reduced cell proliferation activity in the tumor mass although the normal-appearing parts of the gut epithelia were positively responsive to proliferation signals by EGF-EcN (Figure 12B). Compared with the prominent expression of Ki-67 in the cryptic parts, in the normal-appearing parts, adenoma displayed a relatively even level of expression of the protein throughout the epithelial tumor tissue. Moreover, expression of Sox-9 as a self-renewal and proliferation marker of colorectal cancer (34) was also downregulated in the tumor mass by the recombinant probiotic bacteria (Figure 12C). Despite extensive activation of an EGFR-linked signal, cell growth-related fingerprints in the tumor mass faded with EGF-EcN colonization. In spite of the growth factor-linked signaling stimulation by EGF-EcN, the mass growth responses were attenuated in the adenoma of the gut mucosa. To determine the mechanistic implications, we revisited the barrier-protective actions of EGF-EcN in the colitis model. Similar to EGF-EcN-enhanced barrier integrity in mice with colitis, the recombinant 
A AOM $10 \mathrm{mg} / \mathrm{kg}$ i.p inoculation inoculation inoculation

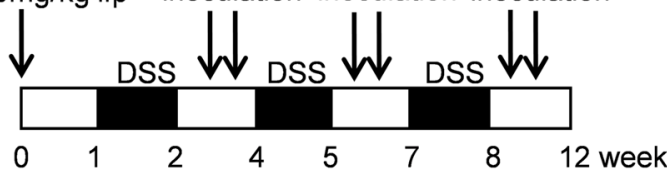

B

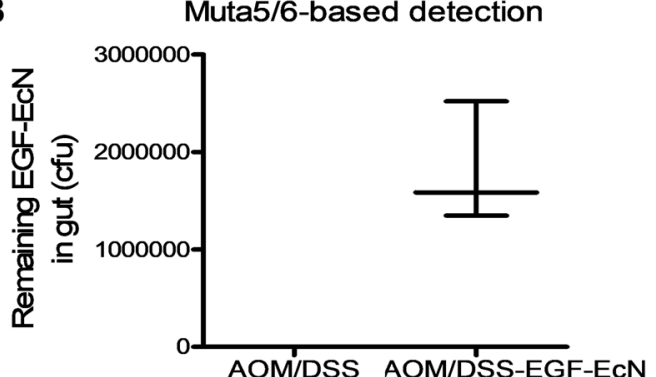

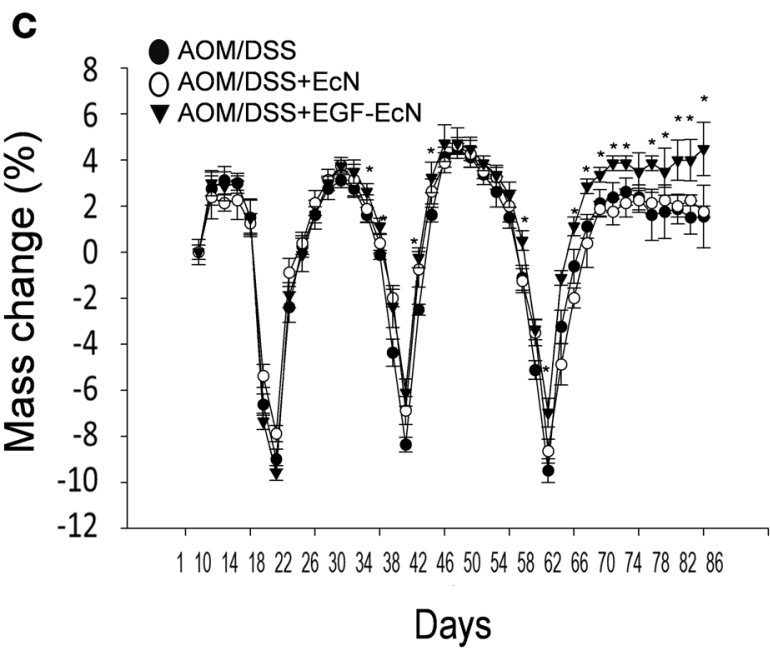

AOM/DSS+EGF-ECN
D

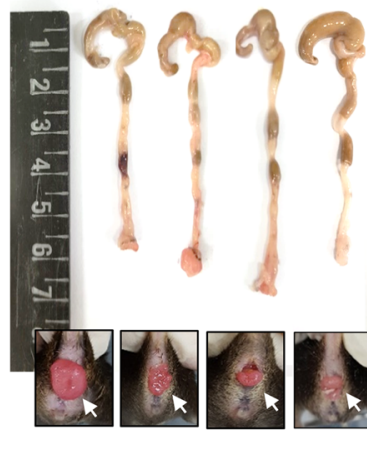

E
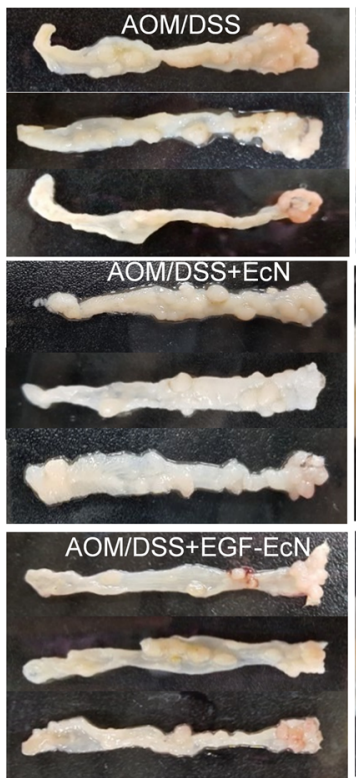

AOM/DSS+EcN
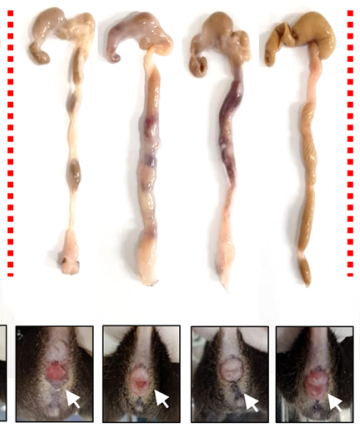

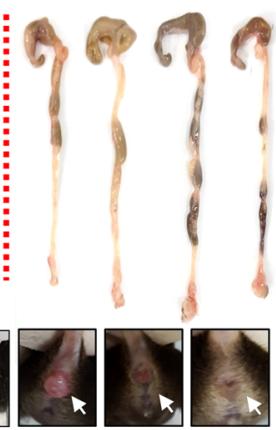

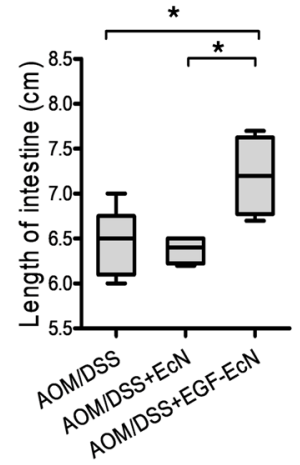

$\mathbf{F}$
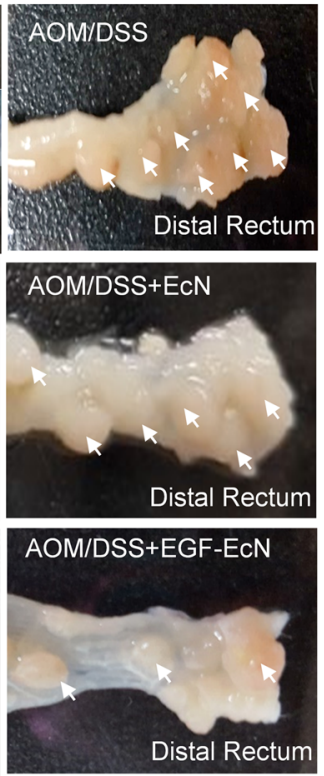
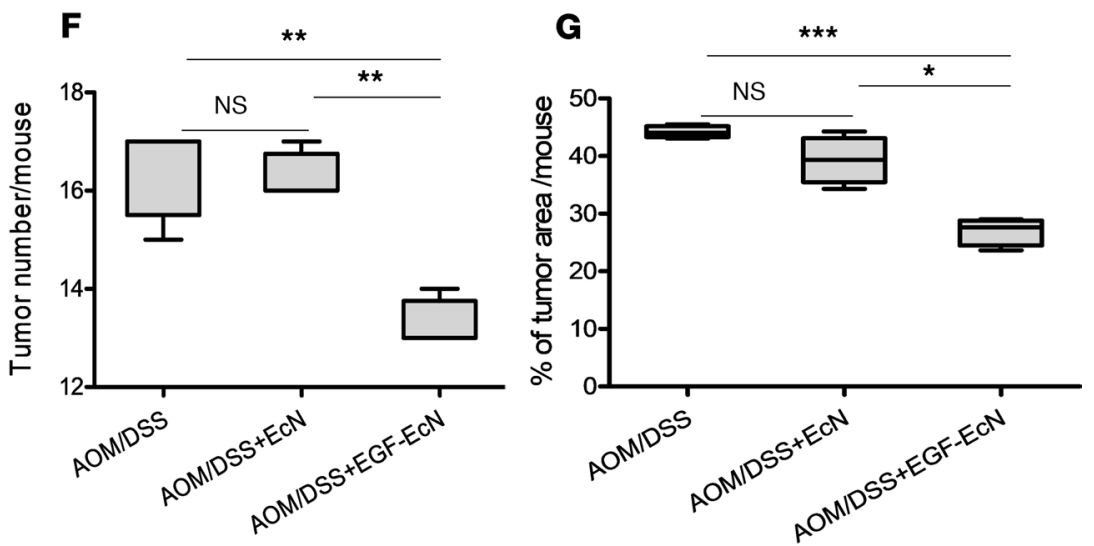

Figure 10. Actions of EGF-EcN in colitis-associated tumorigenesis. Tumors were induced in C57BL/6 mice by using AOM/DSS. (A) Experimental procedure of AOM/DSS exposure along with EcN and EGF-EcN treatment $(n=10-20)$. (B) Stool samples from mice were collected on the final day of the 12-week exposure as indicated in the treatment regime (A). Colonized bacteria were estimated based on PCR with EcN-specific primers for Muta5/6. (C) Mouse body weight change was monitored at indicated times after AOM injection. The asterisks in the graph represent significant differences from mass changes in the AOM/DSS treatment group at each time point ( ${ }^{*} P<0.05$ using 2-tailed, unpaired Student's $t$ test). (D) Colon length was compared. Results of quantitative analyses of colon length (upper) and symptoms in the anus (lower) are demonstrated. (E) Luminal parts of the colorectum used to detect adenomatous polyps (left) and a magnified illustration of the distal rectum (right). (F and $\mathbf{G}$ ) Tumor number (F) and tumor area (G) based on the observation presented in $\mathbf{E}$. The asterisks in box-andwhisker plots ( $\min$ to $\max )(\mathbf{D}, \mathbf{F}$, and $\mathbf{G}$ ) represent significant differences between 2 groups ( ${ }^{*} P<0.05 ;{ }^{* *} P<0.01 ;{ }^{* * *} P<0.001$; ns using 2-tailed, unpaired Student's $t$ test). 
A

Tumor-size:

$\square$

$>0.9 \mathrm{~mm}^{2}$ $<0.9 \mathrm{~mm}^{2}$

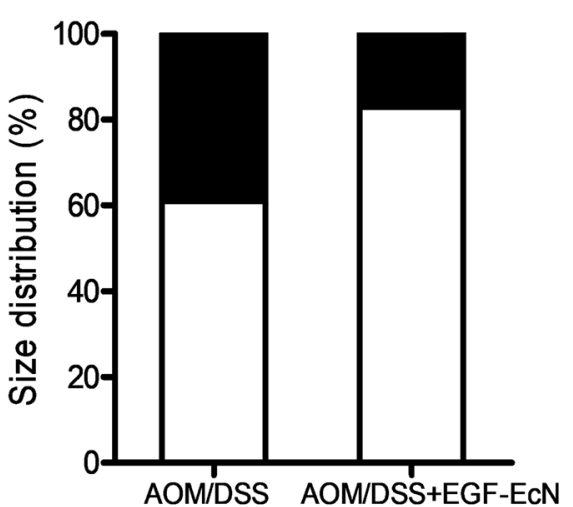

C
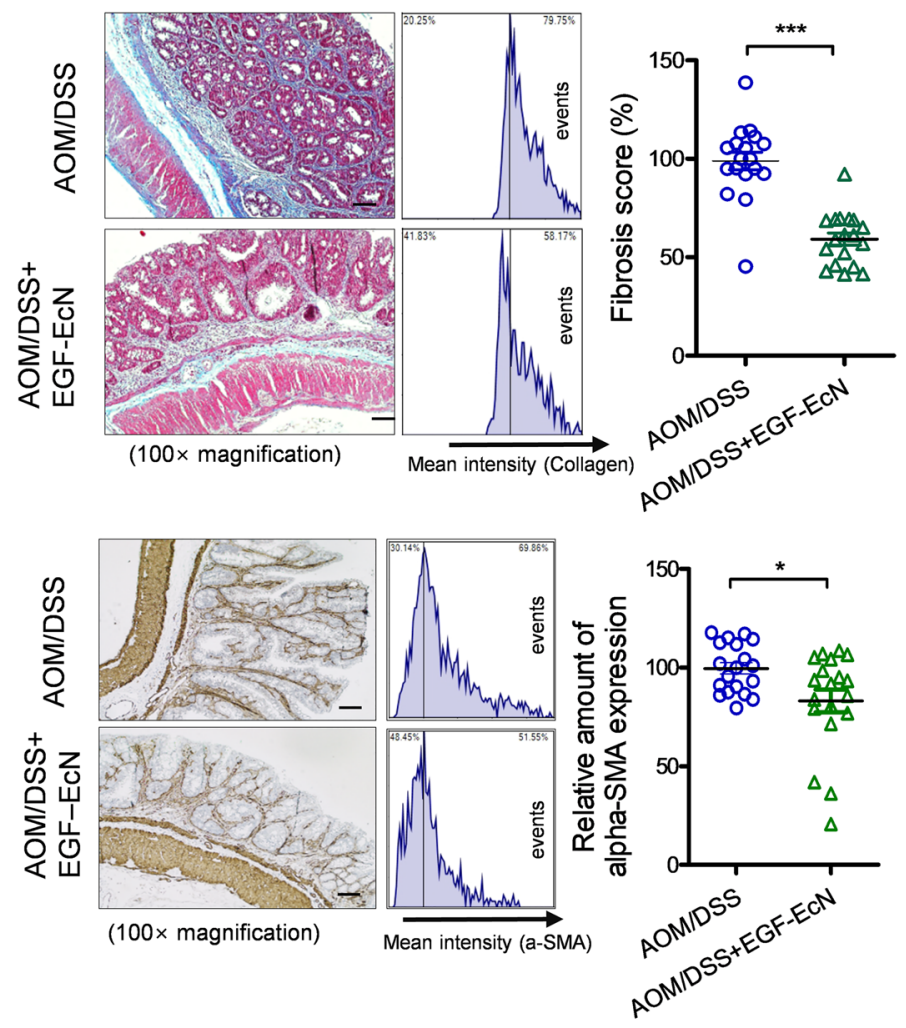

B

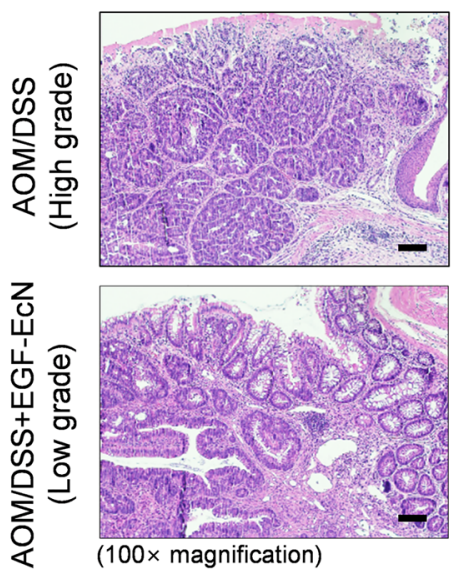

$53.3 \%$

$46.7 \%$

Figure 11. (A) Tumor size distribution based on histological observations ( ${ }^{*} P<0.05$ using 2 -tailed, unpaired Student's $t$ test). (B) H\&E staining of tumor mass (original magnification, $\times 100$ ) and the frequencies of high- and low-grade dysplasia (right graphs). Scale bar: $100 \mu \mathrm{m}$. (C and D) The extent of tissue fibrosis in tumor mass of the AOM/DSS-induced cancer model was determined by examining Masson's trichrome stain (C, original magnification, $\times 100$ ) or IHC for alpha-smooth muscle actin ( $\alpha$-SMA) (D, original magnification, $\times 100)$ results. Scale bar: $100 \mu \mathrm{m}$. Each histogram represents events with increasing collagen (C) or $\alpha$-SMA (D) levels. A quantitative comparison is shown in the right graph $\left({ }^{*} P<0.05 ;{ }^{* *} P<0.01 ;{ }^{* *} P\right.$ $<0.001$ using 2-tailed, unpaired Student's $t$ test).

probiotic treatment enhanced mucus production in both the normal-appearing parts and the tumor mass (Figure 13A). Moreover, the apical integrity of the epithelial lining of the gut was improved by EGF-EcN in the colitis-associated cancer model (Figure 13B). Close observation of F-actin indicated that the tumor-inducing treatment (AOM/DSS) severely disrupted F-actin-based apical projections in the mouse gut epithelial lining, where quantities of F-actin spread into the basolateral regions and even into the basement membrane (Figure 13B). Treatment with EGF-EcN significantly prevented disruption of the apical arrangements in the gut epithelial layer. Based on a transcriptomic analysis using clinical data sets of patients with IBD, EGFR expression was positively correlated with the expression of genes for junctional molecules CLDN3 and TJP1 (ZO-1), which are crucial in the maintenance of gut barrier integrity in patients with IBD (Figure 5, A and B). In the present colitis-associated cancer model, EGF-EcN notably elevated ZO-1 expression in both the normal-appearing gut parts and the gut adenoma (Figure 13C). Using the in vitro cancer cell model, EGF-EcN was also shown to induce expressions of ZO-1 and CLDN3, both of which were dependent on the EGFR-linked signaling pathway (Figure 13D). Furthermore, because disruption of the gut barrier 

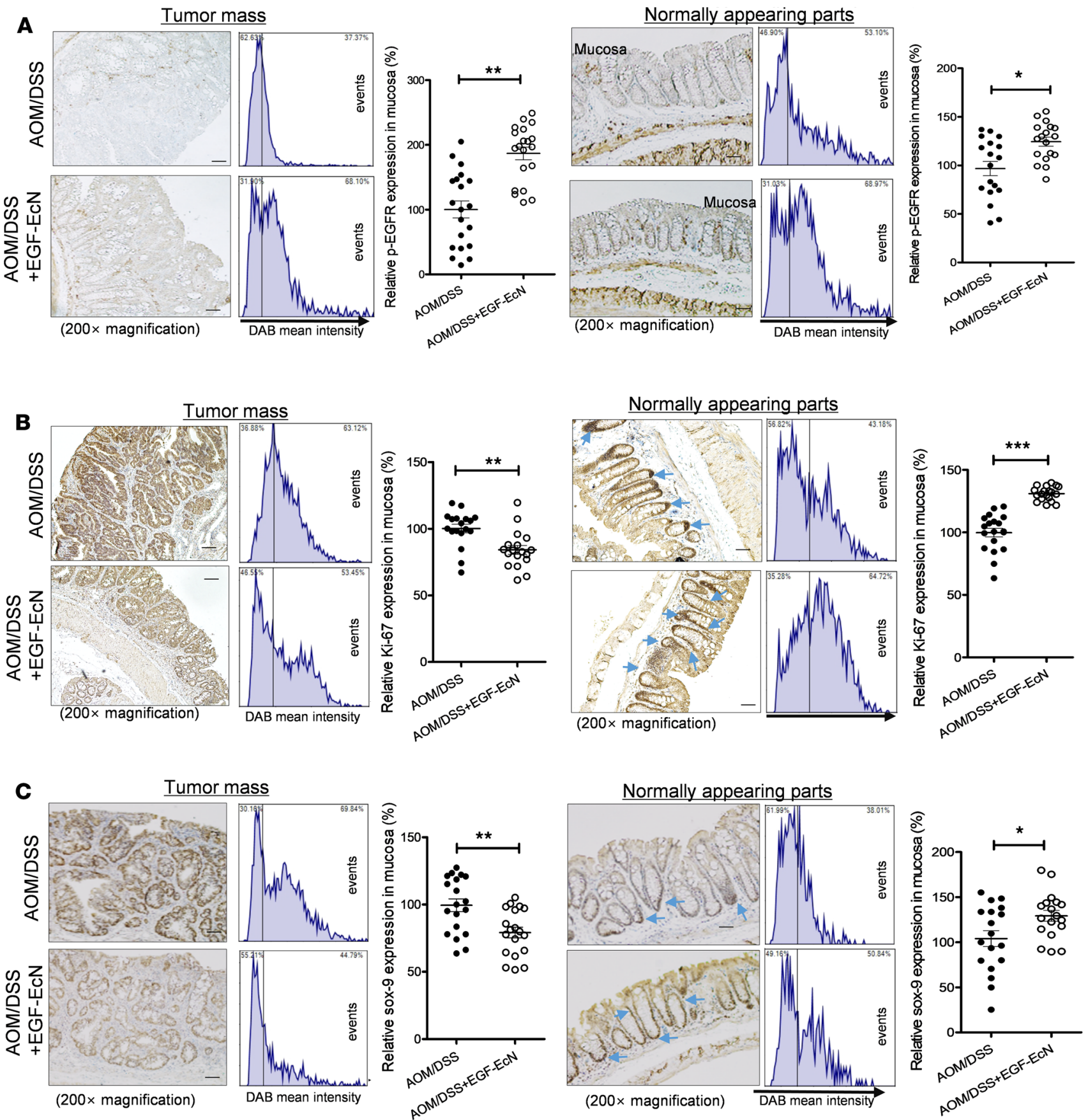

Figure 12. Effects of EGF-EcN on the cell growth signaling in mice with colitis-associated cancer. Tumors were assessed in C57BL/6 mice using AOM/DSS along with EcN and EGF-EcN treatment as depicted in Figure 9A $(n=10-20)$. (A) The expression of active EGFR in colons of the AOM/DSS-induced cancer model was compared (original magnification, $\times 200$ ). Scale bar: $100 \mu \mathrm{m}$. Relative density of p-EGFR was measured by using HistoQuest tissue analysis software. (B) The extent of cell proliferation in colons of the AOM/DSS-induced cancer model was determined by measuring the expression of Ki-67 in the tumor mass (original magnification, $\times 200$ ). Scale bar: $100 \mu \mathrm{m}$. Blue arrows indicate high expressions in the cryptic parts. (C) Sox-9 expression in colons of the AOM/DSS-induced cancer model was determined (original magnification, $\times 200$ ). Scale bar: $200 \mu \mathrm{m}$. Each histogram represents events at an increasing DAB level. A quantitative comparison is shown in the right graph $\left({ }^{*} P<0.05 ;{ }^{*} P<0.01 ;{ }^{* * *} P<0.001\right.$ using 2-tailed, unpaired Student's $t$ test).

facilitates exposure to gut bacteria and subsequent proinflammatory stimulation, EGF-EcN-improved barrier integrity may suppress an inflammatory insult from the gut lumen. EGF-EcN treatment significantly suppressed neutrophil infiltration in the mucosa of mice with colitis-associated colorectal cancers (Figure 13E). Moreover, proinflammatory cytokines, such as interleukin 6 (IL-6) and tumor necrosis factor- $\alpha$, were measured in the blood circulation in the present cancer model. In particular, EGF-EcN treatment led to a 

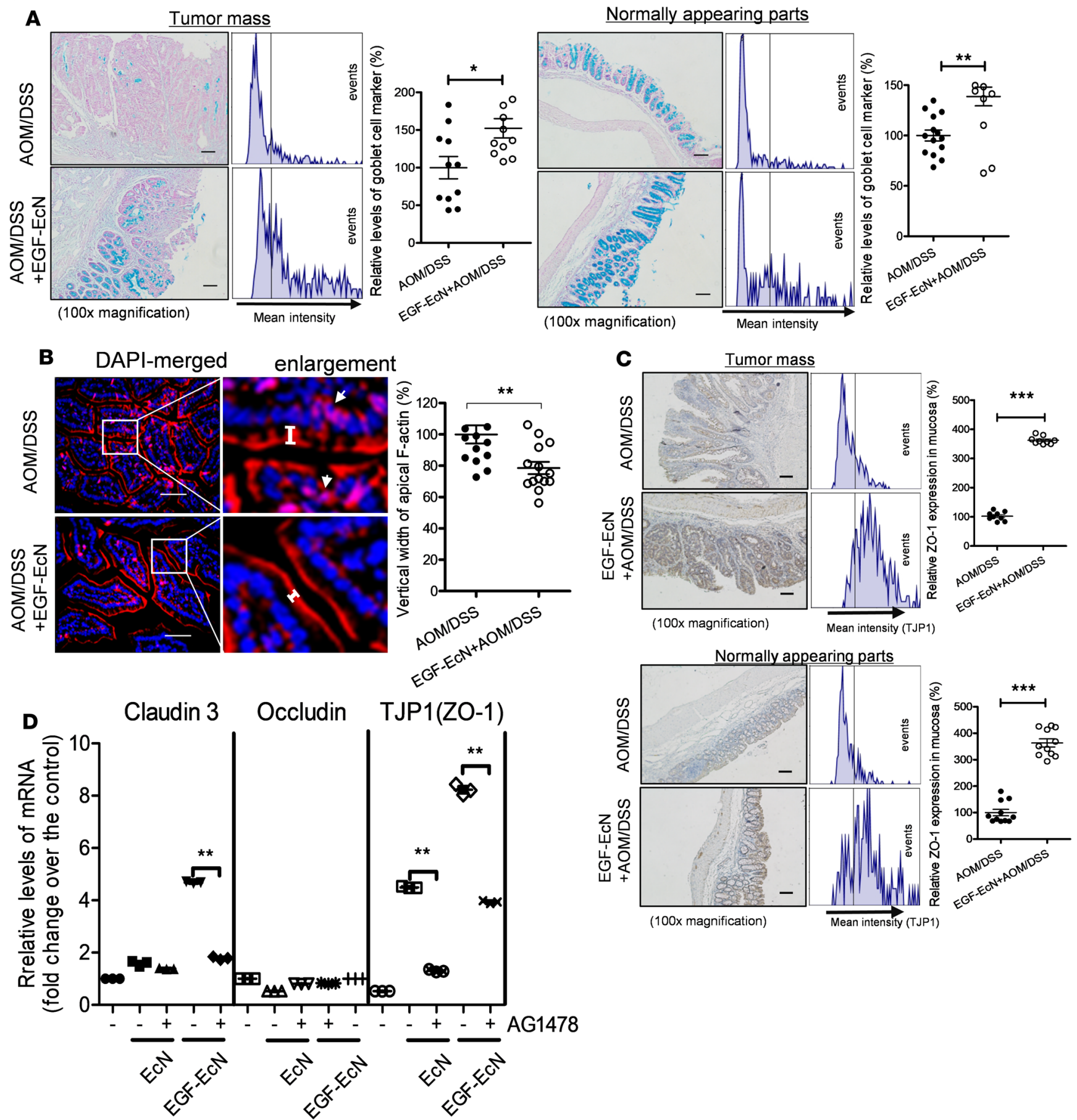

E Colon adenoma

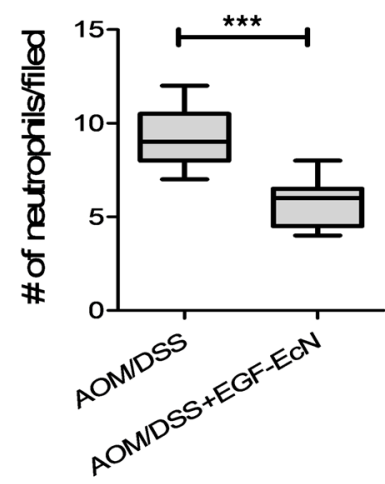

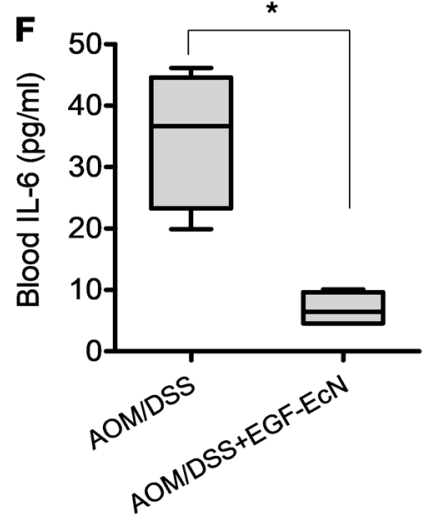

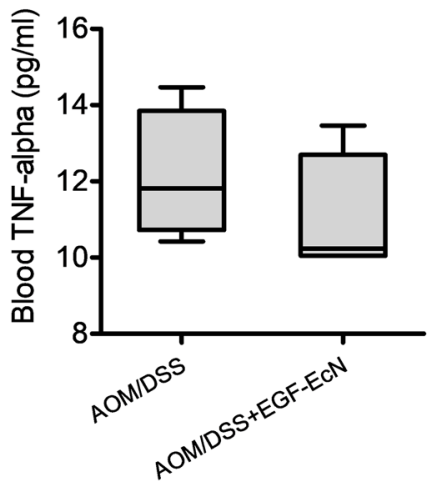


Figure 13. Barrier regulation by EGF-EcN in colitis-associated cancer. Tumors were assessed in C57BL/6 mice using AOM/DSS along with EcN and EGF-EcN treatment ( $n=10-20)$ (A-C, E, F). (A) Colons (tumor mass and normal parts) were isolated for analysis of goblet cells and mucin production and stained with Alcian blue (original magnification, $\times 100$. Scale bar: $100 \mu \mathrm{m}$ ). (B) Gut epithelia (tumor mass and normal parts) were stained with DAPI (blue) and phalloidin-TRITC (red) to stain nucleic acid and F-actin (original magnification, $\times 400$; inset magnification, $\times 8000$ ). Scale bar: $100 \mu \mathrm{m}$. (C) Z0-1 expression in colons of the AOM/DSS-induced cancer model was determined (original magnification, $\times 100)$. Scale bar: $100 \mu \mathrm{m}$. Each histogram represents events at an increasing DAB level. A quantitative comparison is shown in the right graph (A-C, ${ }^{*} P<0.05$; ${ }^{* *} P<0.01$; ${ }^{* * *} P<0.001$ using 2-tailed, unpaired Student's $t$ test). (D) HCT-8 cells were pretreated with AG1478 $(10 \mu \mathrm{M})$ for 1 hour and then incubated with EcN or EGF-EcN for 12 hours. Cellular RNA was analyzed using quantitative PCR ( $n=3{ }^{* *} P<0.01$ using 2-tailed, unpaired Student's $t$ test). (E and $\left.\mathbf{F}\right)$ Neutrophil infiltration (E) in the tumor mass and serum cytokine levels $(\mathbf{F})$ in the AOM/DSS-induced murine tumor model ( ${ }^{*} P<0.05$; ${ }^{* * *} P<0.001$ using 2 -tailed, unpaired Student's $t$ test).

notable decrease in serum IL-6 production in response to tumor formation (Figure 13F). Taken together, EGF-EcN treatment did not aggravate the progression of colitis-associated cancer and even improved gut barrier integrity and decreased inflammatory readouts, which could relieve concerns about the chronic use of mucosal EGF by probiotic bacteria-based delivery.

\section{Discussion}

In the present study, we improved the quality of the $\mathrm{EcN}$ probiotic factory by introducing secretion systems for therapeutic proteins in insulted gut epithelia. Preventive and postinjury treatment with EGF-EcN showed persistent colonization (even after more than 1 month) and alleviated ulcerative injuries in murine small and large intestines by stimulating epithelial restitution. Although $\mathrm{EcN}$ itself exerted some protective effects on the insulted intestine, secreted EGF enhanced the wound healing actions via elevation of the integrity of the gut epithelial and mucosal barrier in mice with colitis or colitis-associated tumor.

Although EGF could be beneficial in the treatment of acute ulcerative injuries by inducing mucosal healing and minimizing luminal bacteria-induced inflammation, systemic use of EGF would obviously be limited by concern about its ability to promote abnormal epithelial growth and tumorigenesis. This concern may limit the duration of topical EGF therapies for the treatment of ulcerative diseases, including IBD. In this study, short-term treatment using topical EGF appeared to achieve clinical remission; moreover, repeated treatment with EGF-EcN did not promote dysplastic growth in the setting of chronic epithelial inflammation and AOM/ DSS-induced tumorigenesis, even suppressing mitogenic (cell growth-promoting), fibrogenic, and inflammatory levels. In agreement with our present results, EGFR inhibition can retard colitis-associated cancer (CAC), although EGFR monoclonal antibodies or EGFR inhibitors are promising interventions in the treatment of colorectal cancer (35). In particular, loss of EGFR signaling increases tumor progression in the AOM/DSSinduced CAC model because EGFR actually acts as a tumor suppressor, likely through stimulation of epithelial regeneration and thus reducing mucosal inflammatory stimulation (35). In the present experimental niche, EGF-EcN-exposed normal epithelia show wound-promoting activities whereas EGF-EcN retarded tumor mass growth potency. EGF-EcN-induced maintenance of epithelial wound healing and integrity under malignant insults could be beneficial because it may prevent barrier disruption-induced inflammatory insults. Moreover, in the CAC model, EGF-EcN suppressed levels of mitogenic readouts (Ki-67 or Sox-9) in the tumor mass while elevating their expression in the normal parts. This differential outcome can be explained in terms of gut barrier integrity. EGF-EcN enhanced barrier integrity via epithelial cell junctions, apical projection of cytoskeleton, and mucus secretion, all of which may limit excessive access of gut microbes and subsequent proinflammatory insult. Mucosal inflammatory cells produce a wide array of reactive oxygen and nitrogen species, various cytokines, and mitogenic growth factors that can favor tumor growth and sustainability (36). Therefore, the barrier-protective actions of recombinant bacteria are expected to prevent microbe-induced inflammation and subsequent formation of growth-promoting tumor niches. The severely injured parts or adenomas can allow more microbial access from the gut lumen than that allowed by normal or intact parts, which keep their barrier integrity. Therefore, mitogenic stimulation would be higher in injured or transformed parts than in normal regions. Compared with the mitogenic signals related to gut bacteria-induced inflammatory insult, EGF-EcN-induced cell growth signals would be meager in the injured parts. However, EGF-EcN improved barrier integrity, which efficiently reduced the mitogenic signals associated with gut microbe-induced inflammatory stress. Therefore, cell growth responses (Ki-67 and Sox-9) were attenuated by EGF-EcN in the adenoma whereas the normal-appearing parts were well responsive to EGF-induced growth-promoting signals in the present study. Moreover, recent evidence suggests that EGFR-linked signaling pathways regulate features of cancer stem-like cells and subsequently reduce the clonogenicity of cancer cells (37-39). These observations are consistent with the patterns of Sox-9, as a biomarker of self-renewal and proliferation in the tumor mass, 
observed in the present study. EGF-EcN markedly suppressed the stemness biomarker in the tumor mass while the EGF-induced growth signals were maintained in the normal-appearing parts. Accordingly, EGF-mediated regulation of cancer stem cells can be another beneficial action against chronic epithelial distress, such as epithelial tumorigenesis. These positive previous reports and our current results relieve concerns about the chronic use of EGF-EcN and its prolonged colonizing activity in the gut. However, the differential regulation of cellular growth and stemness between normal and tumor tissues could be further addressed for improved understanding of the action of EGF-EcN.

The results of the present study were in accordance with those of previous studies into the beneficial actions of $\mathrm{EcN}$, which has been evaluated in recent years as a probiotic bacterial treatment of human gastrointestinal ulcerative diseases $(40,41)$. However, its beneficial actions vary depending on the experimental models used (40-43). Although EcN has been used to maintain and induce clinical remission in UC, there is no reported benefit when using $\mathrm{EcN}$ as an add-on treatment to conventional antibiotic therapies for active UC (42). EcN treatment without a previous antibiotic cure resulted in fewer patients reaching clinical remission compared with conventional therapies, suggesting that $\mathrm{EcN}$ is efficient in remission and that patients with UC flare-ups could benefit from EcN treatment preceded by antibiotics (42). An extension of this concept is that bacteria can be engineered to generate wound healing modulators, such as EGF. In this study, we present evidence that such engineering may improve the benefit of natural EcN, which has been somewhat controversial in clinical trials. In addition to $\mathrm{EGF}-\mathrm{EcN}$, treatment with $\mathrm{EcN}$ itself was also partly protective against ulcerative injuries. Moreover, the inhibitor of EGFR tyrosine kinase attenuated the actions of EcN against ulcerative injuries in the murine model. Mechanistically, in addition to EGF itself as a strong canonical ligand to EGFR, other ligands, such as bacterial products, can activate EGFR via noncanonical ligand-induced activation or intracellular signaling crosstalk with their specific receptors $(44,45)$. Therefore, the possibility that bacteria-responsive factors or endogenous components of $\mathrm{EcN}$ can stimulate EGFR-mediated cytoprotection during gut inflammation cannot be excluded. As another gut-protective mediator, probiotic bacteria-derived soluble proteins, such as the p40 protein from Lactobacillus rhamnosus GG, can activate an EGFR-linked signal, which can mediate antiapoptotic and antiinflammatory actions of the probiotic bacteria (46). Moreover, EcN itself can act favorably by enhancing the mucosal barrier integrity, particularly through upregulation of tight junction-associated proteins in the epithelial layer, although bacterial mediators have not been identified in this regulation (47). However, because these beneficial actions of $\mathrm{EcN}$ on the gut barrier are controversial or marginal, depending on the exposure regime, additional investigations are warranted.

Epithelial integrity via junctional fortification is critical to maintaining active fluid absorption, and its disruption may lead to leaky gut syndrome and noninfective diarrhea because translocation of the commensal bacteria through the disrupted gut barrier may trigger pathological hypersensitive immune activation of underlying lymphocytes. Moreover, EGF secreted from EcN or EGFR-linked signaling can contribute to the maintenance of barrier integrity by modulating tight junction proteins in various intestinal injury models (48-50), indicating the beneficial actions of EGF-EcN against barrier disruption in the present study model. Moreover, EGF-EcN was shown to attenuate goblet cell depletion and mucin suppression in the insulted gut environment in the present study. Signaling activation by EGF has been reported to stimulate goblet cell growth and secretion in the mucosal epithelia $(51,52)$. In addition to the protective actions of the recombinant probiotic bacteria in the colon, EGF-EcN alleviated ulcerative injuries in the small intestine in similar ways. EcN has been shown to act in both the large and small intestines $(53,54)$. In particular, the production of neuroendocrine hormones and drug metabolic enzymes is altered by $\mathrm{EcN}$ in the small intestine, suggesting complicated involvement of $\mathrm{EcN}$-induced epithelial stimulation in the small intestine. Because recombinant $\mathrm{EcN}$ can colonize and act in broad parts of the murine intestine, location-specific and systematic approaches are required to obtain an accurate description of the actions of the recombinant probiotic bacteria for human use. In the present study, constitutive EGF-EcN was shown to be a promising therapeutic biomaterial against ulcerative injuries in the intestine, and the results provide crucial preclinical evidence of its effectiveness for clinical trials. However, careful assessments are needed for safe application in humans because of their more heterogeneous gut luminal environment, including different gut microbiota and diet. The present assessment of EGF-EcN improved the gut luminal interface with the microbiota via improved epithelial and mucosal integrities, which may limit gut bacterial access and microbe-derived inflammatory insult. In the present study, mice with colitis displayed a low $\mathrm{F} / \mathrm{B}$ ratio, an indicator of dysbiosis, but EGF-EcN-induced changes in the bacterial community were marginal. However, the detrimental effect of the changed microbiota composition can be minimized because of the tighter and thicker barrier facilitated by the recombinant probiotic traveler. 


\section{Methods}

Supplemental Methods are available online with this article.

Cell culture. The human intestinal epithelial cell line HCT-8 was purchased from the American Type Culture Collection. The cells were maintained in RPMI 1640 medium (Welgene) containing 10\% (v/v) heat-inactivated fetal bovine serum (FBS; Wellgene), $50 \mathrm{U} / \mathrm{ml}$ penicillin, and $50 \mu \mathrm{g} / \mathrm{ml}$ streptomycin (Welgene). Cells were maintained at $37^{\circ} \mathrm{C}$ in humidified $5 \% \mathrm{CO}_{2}$ incubators. The cell numbers and viability were assessed by the exclusion of trypan blue dye (Merck) using a hemocytometer.

Bacterial growth conditions and in vivo application. Bacterial strains used in this study include E. coli K12 and $\mathrm{EcN}_{\mathrm{C}}$. The $\mathrm{EcN}$ was provided by Ardeypharm GmbH. To secrete human EGF by ABC transporter, human EGF was fused with LARD. We inserted human EGF-LARD and ABC transporter of E. chrysanthemi, prtDEF, in the genome of $\mathrm{EcN}$ by using $\mathrm{pKOV}$, a gene replacement vector. Each strain was routinely grown at $37^{\circ} \mathrm{C}$ in Luria-Bertani (LB) medium to an optical density at $600 \mathrm{~nm}$ of approximately 1, washed by centrifugation (168 $\mathrm{g}, 5$ minutes), and resuspended in serum-free RPMI medium. For probiotic treatment, mice received 2 intragastric gavages with $0.2 \mathrm{ml}$ of a suspension containing $9.0 \log _{10} \mathrm{CFU} / \mathrm{ml} \mathrm{of}$ $\mathrm{EcN}$ for 10 days before colitis induction. To assess bacterial colonization in the gut, recombinant bacteria transformed with pGFP-UV plasmid were applied to the mice via gavage, and the green fluorescence in the isolated intestine was detected by using a fluorescence in vivo imaging system (FM03-B, NeoScience).

Colonization activities of EGF-EcN in the gut of $C$. elegans. Bacterial strains were cultured in LB broth containing $1 \%$ mannose (EPEC) or $50 \mu \mathrm{g} / \mathrm{ml}$ streptomycin (OP50). Wild-type $C$. elegans (N2) obtained from the Caenorhabditis Genetics Center (Minneapolis, Minnesota, USA) were maintained on Nematode Growth Medium (NGM; $50 \mathrm{mM} \mathrm{NaCl}, 1.7 \%$ Agar, 0.25\% peptone, $1 \mathrm{mM} \mathrm{CaCl}$, $5 \mu \mathrm{g} / \mathrm{ml}$ of cholesterol, $1 \mathrm{mM} \mathrm{MgSO} 4$, and $25 \mathrm{mM} \mathrm{KPO} 4$ in $\mathrm{dH}_{2} \mathrm{O}$; made in house). L4 stage nematodes were inoculated for 24 hours on E. coli OP50- or EGF-EcN-spread NGM plates that included $50 \mu \mathrm{M}$ Fluorodeoxyuridine (FUdR; MilliporeSigma). The pretreated worms were infected with GFP-expressing EPEC for 24 hours and were then incubated in a levamisole-M9 solution containing $100 \mu \mathrm{g} / \mathrm{ml}$ gentamycin (MilliporeSigma) for 45 minutes to remove external bacteria before analysis

Analysis of colon or ileal $m R N A$ of patients with IBD. Three human intestinal tissue data sets were obtained from the gene expression array of patients with IBD (Sleiman's, GEO ID GSE10616, $n=58$; Vemeire's, GEO ID GSE75214, $n=194$; and Haberman's, GEO ID GSE57945, $n=322$ ). The 3 major clinical subsets of IBD include colon-only CD, iCD, and UC. The Haberman's samples (GEO ID GSE57945, $n=322$ ) were from ileal biopsies obtained during diagnostic colonoscopies of children and adolescents, aged less than 17 years, who presented with IBD-like symptoms. These experiments tested differential colon or ileal gene expressions in these 3 types of IBD relative to those in healthy control samples and the local degree of mucosal inflammation as measured by determining the $\mathrm{CD}$ histological index of severity. Samples were obtained from the most proximal affected segment of the colon or ileum. Sample values were normalized against internal controls.

EcN-specific detection using PCR. Feces of mice treated twice with $1 \times 10^{9} \mathrm{EGF}-\mathrm{EcN}$ were collected at 5,30 , and 60 days after gavage. DNA was extracted according to the indicated method by using a stool DNA mini kit (GeneAll). Supernatant from $100 \mathrm{mg}$ of stool in PBS was collected for DNA isolation after homogenization and centrifugation. The isolated DNA was used as a template and detected using $2 \mathrm{EcN}$-specific primers (Muta5/6 and Muta7/8). The following primers were used for PCR: Muta5/6, 5'-AACTGTGAAGCGATGAACCC-3' and 5'-GGACTGTTCAGAGAGCTATC-3'; Muta7/8, 5'-GACCAAGCGATAACCGGATG-3' and 5'-GTGAGATGATGGCCACGATT-3'.

Murine intestinal injury. The mice were purchased from The Jackson Laboratory and allowed to acclimate for 7 days. All mice were individually housed in ventilated cages with free access to feed and water and acclimated to standard laboratory conditions (12-hour light/12-hour dark cycle, temperature $22^{\circ} \mathrm{C} \pm$ $\left.1^{\circ} \mathrm{C}\right)$. For the DSS model, female 8-week-old C57BL/6 mice were observed because male mice are more sensitive to DSS than female mice and tend to show higher mortality when acutely exposed to DSS (55, 56). To induce large intestinal injury, mice were administered 3\% (w/v) DSS (36,000-50,000 Da; MP Biomedical) ad libitum in drinking water for 5 days. Control mice received regular drinking water during the entire experiment. All animals were sacrificed under deep ether anesthesia. For the indomethacin model, the male C57BL/ 6 mice were 10 weeks old and weighed $20-23 \mathrm{~g}$. To induce small intestinal injury, the mice were given indomethacin ( $30 \mathrm{mg} / \mathrm{kg}$, oral gavage) after fasting for 18 hours. Twenty-four hours later, they were assessed. For the AOM/DSS-induced colon tumorigenesis model, 7-week-old female C57BL/6 mice were given a single intraperitoneal injection of $\mathrm{AOM}$ at a dose of $10 \mathrm{mg} / \mathrm{kg}$, followed by 3 cycles of 
2.5\% DSS for 7 days in drinking water ad libitum with 2 weeks of normal water between each cycle. Mice were sacrificed 12 weeks after the AOM injection.

Histological scoring of colitis or small intestinal injury. H\&E staining was performed using standard procedures. Scoring of disease severity was based on the following 4 parameters as described in a previous report (57). Severity of inflammation was scored as follows: 0 , rare inflammatory cells in the lamina propria; 1 , increased numbers of granulocytes in the lamina propria; 2, confluence of inflammatory cells extending into the submucosa; 3 , transmural extension of the inflammatory infiltrate. Crypt damage was scored as follows: 0 , intact crypts; 1 , loss of the basal half; 2 , entire crypt loss; 3 , confluent erosion. Ulceration was scored as follows: 0 , absence of ulcer; 1,1 or 2 foci of ulcerations; 2, 3 or 4 foci of ulcerations; 3 , confluent or extensive ulceration. Edema was scored as follows: 0, absence of edema; 1, mild; 2, moderate; 3, severe.

Western blot. The intestine was rinsed with ice-cold PBS and cut into small pieces $(1 \mathrm{~cm})$. Tissue samples were homogenized in a protein lysis buffer $(10 \mathrm{mM}$ Tris- $\mathrm{HCl}, \mathrm{pH} 7.4 ; 1 \mathrm{mM}$ sodium orthovanadate; $1 \%$ SDS) using TissueLyser (Qiagen) and stainless steel beads (5 mm). All samples were centrifuged at $140 \mathrm{~g}$ for 15 minutes at $4^{\circ} \mathrm{C}$, after which the supernatant was collected. The process of Western blot analysis was described previously (57).

IHC. Tissue samples were dehydrated, embedded in paraffin, and cut into 5 - $\mu \mathrm{m}$ sections for IHC analysis. Tissue sections $(5 \mu \mathrm{m})$ were blocked with 5\% FBS in PBS with 0.1\% Tween 20 (PBST), incubated in a 1:250 dilution of the primary antibodies overnight at $4^{\circ} \mathrm{C}$, and repeatedly washed using PBST. Antibodies include: rabbit polyclonal anti-mouse p-EGFR (Tyr1068) antibody (2234, Cell Signaling Technology, rabbit polyclonal anti-mouse Sox-9 antibody (SC-20095, Santa Cruz Biotechnology Inc.), rabbit polyclonal anti-mouse claudin-3 antibody (A2946, ABclonal Technology), rat monoclonal anti-mouse TJP1 antibody (SC-33725, Santa Cruz Biotechnology), and rabbit polyclonal anti-mouse cleaved caspase-3 (ASP175) antibody (9661, Cell Signaling Technology). Samples were incubated with horseradish peroxidase-conjugated secondary antibody (goat polyclonal anti-rabbit IgG [ADI-SAB-300, Enzo]) and goat polyclonal anti-rat IgG heavy and light chain antibody (A110-105P, Bethyl Laboratories). The bound antibodies were then identified using freshly prepared substrate buffer (0.05\% DAB [MilliporeSigma], 0.015\% $\mathrm{H}_{2} \mathrm{O}_{2}$ in PBS) for 2 minutes. For immunofluorescence detection, the secondary antibodies were goat anti-mouse 488 (1/250, Bethyl Laboratories) and Alexa Fluor 594-labeled anti-rabbit antibodies (1/250, Life Technology), and the sections were mounted with DAPI mounting solution (blue). Stained sections were subsequently examined at various magnifications using a fluorescence microscope (Nikon). To analyze the proportion of positively stained cells in the tissue, at least 4 representative areas were measured by computer-assisted analysis using HistoQuest image analysis software version 4.0 (TissueGnostics). The pixels of images viewed in this software are converted to grayscale and assigned an arbitrary number relating to staining intensity. HistoQuest image analysis software differentiates hematoxylin-positive and DAB-positive cells or areas that are recorded as events (cells/nuclei) or area of staining. Results are presented as histograms to which staining area or intensity cutoffs can be applied to differentiate between cell populations. The results allow determination of how many cells express how much of a given marker. Values were optimized by counting the numbers of DAB-positive events and hematoxylin-positive events. The graphs statistically compare the values among groups.

BrdU staining. The labeling index of proliferated cells in the intestine was determined by assaying BrdU (BD Immunocytometry Systems) staining levels. Briefly, nonfasting mice received an intraperitoneal injection of $20 \mathrm{mg} / \mathrm{kg}$ body weight of BrdU, and intestinal cells were analyzed 1 hour later. The BrdU-labeled cells were identified by the presence of dark pigment over their nuclei. To analyze the labeling index of proliferated cells, we counted the number of BrdU-labeled cells among the intestinal epithelial cells in 10 crypts.

Masson's trichrome staining. Masson's trichrome staining was performed to examine the compositional change of connective tissues and used according to the manufacturer's instructions (Abcam). Connective tissues, including collagen, were stained blue; nuclei were stained dark red/purple; and cytoplasm or muscle fibers were stained red/pink.

Cytokine analysis. Blood was retro-orbitally collected to measure the cytokine level. Serum was separated by centrifugation $(1000 \mathrm{~g}, 15$ minutes $)$ and stored at $-80^{\circ} \mathrm{C}$ until measurement. Samples were diluted 1:4 and cytokine levels in serum were measured according to the manufacturers' instructions for a Bio-Plex Pro assay kit (Bio-Rad) using a Luminex 200 system (Thermo Fisher Scientific).

Fluorometric assay of FITC-conjugated dextran in vivo. Intestinal permeability was evaluated in different groups of mice by using an orally administered permeability marker, FITC-conjugated dextran, dissolved in water ( $4 \mathrm{kDa}, 50 \mathrm{mg} / \mathrm{ml}$, MilliporeSigma). Blood serum was collected 3 hours later. 
The serum concentration of FITC-conjugated dextran was determined by using a plate reader (SpectraMAX M2e, Molecular Devices). The sample emission spectrum (535 nm) was recorded after excitation at $490 \mathrm{~nm}$. The concentration of FITC-conjugated dextran in serum was determined by applying an FITC-dextran standard curve.

Statistics. Statistical analyses were performed using GraphPad Prism version 5.01. For comparative analysis of 2 groups of data, Student's $t$ test was performed. For comparative analysis of multiple groups, data were subjected to 1-way ANOVA with Newman-Keuls method as a post hoc ANOVA assessment. For 2-gene correlation coefficient $(R)$ determination in IBD-based data sets, Pearson's correlation analysis was performed.

Study approval. This animal study was approved by the Pusan National University Institutional Animal Care and Use Committee (Busan, South Korea) (PNU-2015-0786), and the methods were carried out in accordance with the Declaration of Helsinki and with the Guide for the Care and Use of Laboratory Animals (National Academies Press, 2011) as adopted and promulgated by the US National Institutes of Health.

\section{Author contributions}

Project design and hypotheses were developed by YM. MY, JK, and JHA conducted the experiments and analyzed the data. JK was in charge of parts of revision experiments and all issues of graphic optimization. YM prepared the manuscript and supervised the overall project.

\section{Acknowledgments}

This research was supported by the Basic Science Research Program through the National Research Foundation of Korea funded by the Ministry of Education (2018R1D1A3B05041889). We appreciate early experimental assistance by Hye Jin Choi and Kee Hun Do (Pusan national university, Yangsan, Korea).

Address correspondence to: Yuseok Moon, Department of Biomedical Sciences, Pusan National University, Yangsan, South Korea 50612. Phone: 82.51.510.8094; Email: moon@pnu.edu.

1. Lacy ER. Prostaglandins and histological changes in the gastric mucosa. Dig Dis Sci. 1985;30(Suppl 11):83S-94S.

2. Yeomans ND, Skeljo MV. Repair and healing of established gastric mucosal injury. J Clin Gastroenterol. 1991;13(Suppl 1):S37-S41.

3. Medema JP, Vermeulen L. Microenvironmental regulation of stem cells in intestinal homeostasis and cancer. Nature. 2011;474(7351):318-326.

4. Roda G, et al. Intestinal epithelial cells in inflammatory bowel diseases. World J Gastroenterol. 2010;16(34):4264-4271.

5. Lacy ER, Ito S. Rapid epithelial restitution of the rat gastric mucosa after ethanol injury. Lab Invest. 1984;51(5):573-583.

6. Jones MK, Tomikawa M, Mohajer B, Tarnawski AS. Gastrointestinal mucosal regeneration: role of growth factors. Front Biosci. 1999;4:D303-D309.

7. Tarnawski A. Molecular mechanisms of ulcer healing. Drug News Perspect. 2000;13(3):158-168.

8. Konturek SJ, Brzozowski T, Majka J, Dembinski A, Slomiany A, Slomiany BL. Transforming growth factor alpha and epidermal growth factor in protection and healing of gastric mucosal injury. Scand J Gastroenterol. 1992;27(8):649-655

9. Pai R, Tarnawski A. Signal transduction cascades triggered by EGF receptor activation: relevance to gastric injury repair and ulcer healing. Dig Dis Sci. 1998;43(Supp1 9):14S-22S.

10. Itoh M, Matsuo Y. Gastric ulcer treatment with intravenous human epidermal growth factor: a double-blind controlled clinical study. J Gastroenterol Hepatol. 1994;9(Suppl 1):S78-S83.

11. Haedo W, et al. Oral human recombinant epidermal growth factor in the treatment of patients with duodenal ulcer. Rev Esp Enferm Dig. 1996;88(6):409-418.

12. Palomino A, et al. A multicenter, randomized, double-blind clinical trial examining the effect of oral human recombinant epidermal growth factor on the healing of duodenal ulcers. Scand J Gastroenterol. 2000;35(10):1016-1022.

13. Modi NB. Pharmacokinetics and pharmacodynamics of recombinant proteins and peptides. J Control Release. 1994;29(3):269-281.

14. Horn N, Carvalho AL, Overweg K, Wegmann U, Carding SR, Stentz R. A novel tightly regulated gene expression system for the human intestinal symbiont Bacteroides thetaiotaomicron. Front Microbiol. 2016; 7:1080.

15. Braat $\mathrm{H}$, et al. A phase I trial with transgenic bacteria expressing interleukin-10 in Crohn's disease. Clin Gastroenterol Hepatol. 2006;4(6):754-759.

16. D'Souza AL, Rajkumar C, Cooke J, Bulpitt CJ. Probiotics in prevention of antibiotic associated diarrhoea: meta-analysis. BMJ. 2002;324(7350):1361.

17. Duncker SC, Lorentz A, Schroeder B, Breves G, Bischoff SC. Effect of orally administered probiotic E. coli strain Nissle 1917 on intestinal mucosal immune cells of healthy young pigs. Vet Immunol Immunopathol. 2006;111(3-4):239-250.

18. Kruis W, Schütz E, Fric P, Fixa B, Judmaier G, Stolte M. Double-blind comparison of an oral Escherichia coli preparation and mesalazine in maintaining remission of ulcerative colitis. Aliment Pharmacol Ther. 1997;11(5):853-858.

19. Do VT, Baird BG, Kockler DR. Probiotics for maintaining remission of ulcerative colitis in adults. Ann Pharmacother. 2010;44(3):565-571

20. Matthes H, Krummenerl T, Giensch M, Wolff C, Schulze J. Clinical trial: probiotic treatment of acute distal ulcerative colitis with rectally administered Escherichia coli Nissle 1917 (EcN). BMC Complement Altern Med. 2010;10:13.

21. Plassmann D, Schulte-Witte H. [Treatment of irritable bowel syndrome with Escherichia coli strain Nissle 1917 (EcN): a retro- 
spective survey]. Med Klin (Munich). 2007;102(11):888-892.

22. Gronbach K, et al. Safety of probiotic Escherichia coli strain Nissle 1917 depends on intestinal microbiota and adaptive immunity of the host. Infect Immun. 2010;78(7):3036-3046.

23. Blum G, Marre R, Hacker J. Properties of Escherichia coli strains of serotype O6. Infection. 1995;23(4):234-236

24. Schulze J, Sonnenborn U. Re.: Oral administration of a certain strain of live Escherichia coli for intestinal disorders? (Infection 23 [1995] 51-54). Infection. 1995;23(3):184-188.

25. Choi HJ, Ahn JH, Park SH, Do KH, Kim J, Moon Y. Enhanced wound healing by recombinant Escherichia coli Nissle 1917 via human epidermal growth factor receptor in human intestinal epithelial cells: therapeutic implication using recombinant probiotics. Infect Immun. 2012;80(3):1079-1087.

26. Kruis W, Chrubasik S, Boehm S, Stange C, Schulze J. A double-blind placebo-controlled trial to study therapeutic effects of probiotic Escherichia coli Nissle 1917 in subgroups of patients with irritable bowel syndrome. Int J Colorectal Dis. 2012;27(4):467-474.

27. Tannock GW, et al. Testing probiotic strain Escherichia coli Nissle 1917 (Mutaflor) for its ability to reduce carriage of multidrug-resistant E. coli by elderly residents in long-term care facilities. J Med Microbiol. 2011;60(Pt 3):366-370.

28. Nadkarni MA, Martin FE, Jacques NA, Hunter N. Determination of bacterial load by real-time PCR using a broad-range (universal) probe and primers set. Microbiology (Reading, Engl). 2002;148(Pt 1):257-266.

29. Chan FK. Primer: managing NSAID-induced ulcer complications--balancing gastrointestinal and cardiovascular risks. Nat Clin Pract Gastroenterol Hepatol. 2006;3(10):563-573.

30. Morris AJ, MacKenzie JF. Small-bowel enteroscopy and NSAID ulceration. Lancet. 1991;337(8756):1550.

31. Davies NM. Sustained release and enteric coated NSAIDs: are they really GI safe? J Pharm Pharm Sci. 1999;2(1):5-14.

32. Ettarh RR, Carr KE. Structural and morphometric analysis of murine small intestine after indomethacin administration. Scand J Gastroenterol. 1993;28(9):795-802.

33. Ettarh RR, Carr KE. Morphometric analysis of the small intestinal epithelium in the indomethacin-treated mouse. $J$ Anat. 1996;189(Pt 1):51-56.

34. Carrasco-Garcia E, et al. SOX9-regulated cell plasticity in colorectal metastasis is attenuated by rapamycin. Sci Rep. 2016;6:32350

35. Dubé PE, et al. Epidermal growth factor receptor inhibits colitis-associated cancer in mice. J Clin Invest. 2012;122(8):2780-2792.

36. Westbrook AM, Szakmary A, Schiestl RH. Mechanisms of intestinal inflammation and development of associated cancers: lessons learned from mouse models. Mutat Res. 2010;705(1):40-59.

37. Arasada RR, Amann JM, Rahman MA, Huppert SS, Carbone DP. EGFR blockade enriches for lung cancer stem-like cells through Notch3-dependent signaling. Cancer Res. 2014;74(19):5572-5584.

38. $\mathrm{Ma} \mathrm{L}$, et al. Cancer stem-like cell properties are regulated by EGFR/AKT/ $\beta$-catenin signaling and preferentially inhibited by gefitinib in nasopharyngeal carcinoma. FEBS J. 2013;280(9):2027-2041.

39. Sato T, et al. Single Lgr5 stem cells build crypt-villus structures in vitro without a mesenchymal niche. Nature. 2009;459(7244):262-265.

40. Henker J, Müller S, Laass MW, Schreiner A, Schulze J. Probiotic Escherichia coli Nissle 1917 (EcN) for successful remission maintenance of ulcerative colitis in children and adolescents: an open-label pilot study. Z Gastroenterol. 2008;46(9):874-875.

41. Matthes H, Krummenerl T, Giensch M, Wolff C, Schulze J. Clinical trial: probiotic treatment of acute distal ulcerative colitis with rectally administered Escherichia coli Nissle 1917 (EcN). BMC Complement Altern Med. 2010;10:13.

42. Petersen AM, Mirsepasi H, Halkjær SI, Mortensen EM, Nordgaard-Lassen I, Krogfelt KA. Ciprofloxacin and probiotic Escherichia coli Nissle add-on treatment in active ulcerative colitis: a double-blind randomized placebo controlled clinical trial. J Crohns Colitis. 2014;8(11):1498-1505.

43. Zigra PI, Maipa VE, Alamanos YP. Probiotics and remission of ulcerative colitis: a systematic review. Neth J Med. 2007;65(11):411-418.

44. Kato K, Lillehoj EP, Kim KC. Pseudomonas aeruginosa stimulates tyrosine phosphorylation of and TLR5 association with the MUC1 cytoplasmic tail through EGFR activation. Inflamm Res. 2016;65(3):225-233.

45. Slomiany BL, Slomiany A. Helicobacter pylori-induced gastric mucosal TGF- $\alpha$ ectodomain shedding and EGFR transactivation involves Rac1/p38 MAPK-dependent TACE activation. Inflammopharmacology. 2016;24(1):23-31.

46. Yan F, et al. Colon-specific delivery of a probiotic-derived soluble protein ameliorates intestinal inflammation in mice through an EGFR-dependent mechanism. J Clin Invest. 2011;121(6):2242-2253.

47. Ukena SN, et al. Probiotic Escherichia coli Nissle 1917 inhibits leaky gut by enhancing mucosal integrity. PLoS ONE. 2007;2(12):e1308.

48. Clark JA, Gan H, Samocha AJ, Fox AC, Buchman TG, Coopersmith CM. Enterocyte-specific epidermal growth factor prevents barrier dysfunction and improves mortality in murine peritonitis. Am J Physiol Gastrointest Liver Physiol. 2009;297(3):G471-G479.

49. Raimondi F, et al. Bile acids modulate tight junction structure and barrier function of Caco-2 monolayers via EGFR activation. Am J Physiol Gastrointest Liver Physiol. 2008;294(4):G906-G913.

50. Znalesniak EB, Hoffmann W. Modulation of cell-cell contacts during intestinal restitution in vitro and effects of epidermal growth factor (EGF). Cell Physiol Biochem. 2010;25(4-5):533-542.

51. He M, Lippestad M, Li D, Hodges RR, Utheim TP, Dartt DA. Activation of the EGF receptor by histamine receptor subtypes stimulates mucin secretion in conjunctival goblet cells. Invest Ophthalmol Vis Sci. 2018;59(8):3543-3553.

52. Parker JC, et al. Epidermal growth factor removal or tyrphostin AG1478 treatment reduces goblet cells \& mucus secretion of epithelial cells from asthmatic children using the air-liquid interface model. PLoS ONE. 2015;10(6):e0129546.

53. Matuskova Z, et al. Effects of probiotic Escherichia coli Nissle 1917 on expression of cytochromes P450 along the gastrointestinal tract of male rats. Neuro Endocrinol Lett. 2010;31(Suppl 2):46-50.

54. Nzakizwanayo J, Dedi C, Standen G, Macfarlane WM, Patel BA, Jones BV. Escherichia coli Nissle 1917 enhances bioavailability of serotonin in gut tissues through modulation of synthesis and clearance. Sci Rep. 2015;5:17324

55. Bábíčková J, et al. Sex differences in experimentally induced colitis in mice: a role for estrogens. Inflammation. 2015;38(5):1996-2006.

56. Ding S, et al. Mucosal healing and fibrosis after acute or chronic inflammation in wild type FVB-N mice and C57BL6 procollagen $\alpha 1(\mathrm{I})$-promoter-GFP reporter mice. PLoS ONE. 2012;7(8):e42568.

57. Choi HJ, et al. Early epithelial restitution by nonsteroidal anti-inflammatory drug-activated gene 1 counteracts intestinal ulcer ative injuries. J Immunol. 2016;197(4):1415-1424. 